\title{
On generalized median triangles and tracing orbits
}

\author{
HIROAKI NAKAMURA AND HIROYUKI OGAWA
}

\begin{abstract}
We study generalization of median triangles on the plane with two complex parameters. By specialization of the parameters, we produce periodical motion of a triangle whose vertices trace each other on a common closed orbit.
\end{abstract}

\section{Contents}

1. Introduction

2. Generalized median operators

3. Fourier parameters

4. Reduction of 18 -fold ways of $\mathcal{M}^{\text {wx }}$ yz

5. Shape space and Bényi-Ćurgus lifts

6. Tracing orbits of triangles

Acknowledgement

\section{1. [Introduction}

Given a triangle $\Delta=\triangle A B C$ on a plane, one forms its medial (or midpoint) triangle $\mathcal{S}(\Delta)=$ $\triangle A^{\prime} B^{\prime} C^{\prime}$ which, by definition, is a triangle obtained by joining the midpoints $A^{\prime}, B^{\prime}, C^{\prime}$ of the sides $B C, C A, A B$ respectively. The median triangle $\mathcal{M}(\Delta)=\Delta A^{\prime \prime} B^{\prime \prime} C^{\prime \prime}$ of $\Delta=\triangle A B C$ is a triangle whose three sides are parallel to the three medians $A A^{\prime}, B B^{\prime}, C C^{\prime}$ of $\Delta$. To position $\mathcal{M}(\Delta)$, it is convenient to impose extra condition that $\mathcal{M}(\Delta)$ shares its centroid with $\Delta$ as well as with $\mathcal{S}(\Delta)$. To fix labels of vertices of $\mathcal{M}(\Delta)$, one can set, for example, $\overrightarrow{A A^{\prime}}=\overrightarrow{A^{\prime \prime} B^{\prime \prime}}$, $\overrightarrow{B B^{\prime}}=\overrightarrow{B^{\prime \prime} C^{\prime \prime}}, \overrightarrow{C C^{\prime}}=\overrightarrow{C^{\prime \prime} A^{\prime \prime}}$.

Arithmetic interest on median triangles can be traced back to Euler who found a smallest triangle made of three integer sides and three integer medians: there exists $\triangle A B C$ with $\overline{A B}=$ 136, $\overline{B C}=174, \overline{C A}=170, \overline{A A^{\prime}}=127, \overline{B B^{\prime}}=131$ and $\overline{C C^{\prime}}=158$ (cf. [2]). In recent years, geometrical constructions of nested triangles in more general senses call attentions of researchers (e.g., [1],[9]). In particular, M.Hajja [4] studied a generalization of the above constructions $\mathcal{S}(\Delta)$ and $\mathcal{M}(\Delta)$ by introducing a real parameter $s \in \mathbb{R}$ to replace the midpoints of the sides by more general $(s: 1-s)$-division points. Recently in [8], the former construction for $\mathcal{S}(\Delta)$ was generalized so as to have two complex parameters $\Delta \mapsto \mathcal{S}_{p, q}(\Delta)(p, q \in \mathbb{C}, p q \neq 1)$.

The primary aim of the first part of this paper is, following the line of $[8$, to extend the procedure for $\mathcal{M}(\Delta)$ to a collection of operations of the forms $\Delta \mapsto \mathcal{M}_{p, q}^{\mathrm{wx} / \mathrm{yz}}(\Delta)$ so that the sides of $\mathcal{M}_{p, q}^{\mathrm{wx} / \mathrm{yz}}(\Delta)$ are given by vectors joining vertices of $\Delta$ and of $\mathcal{S}_{p, q}(\Delta)$ in 18-fold ways of label

1991 Mathematics Subject Classification. 51M15; 51N20, 12F05, 43 A32.

This is a pre-print of an article published in Results in Mathematics. The final authenticated version is available online at: https://doi.org/s00025-020-01268-3 
correspondences (See Definition 2.5 below). After studying mutual relations of the 18-fold ways, we will find that only three ways among them are essential. Then, applying the finite Fourier transforms of triangles, we obtain operators $\mathcal{S}\left[\eta, \eta^{\prime}\right]$ and $\mathcal{M}^{\text {wx } / \mathrm{yz}}\left[\eta, \eta^{\prime}\right]$ which behave smoothly with the parameter $\left(\eta, \eta^{\prime}\right)$ running over the full space $\mathbb{C}^{2}$ (the former was already closely studied in $[8]$ ).

In the second part of the present paper, we will study 'dancing' of triangles $\mathcal{S}\left[\eta, \eta^{\prime}\right](\Delta)$ and $\mathcal{M}^{\text {wx } / \mathrm{yz}}\left[\eta, \eta^{\prime}\right](\Delta)$ along with periodical parameters $\left(\eta(t), \eta^{\prime}(t)\right) \in \mathbb{C}^{2}(t \in \mathbb{R} / \mathbb{Z})$. In particular, we search conditions under which the three vertices of a triangle trace one after the other in motion along a single common orbit. Basic examples including "choreographic three bodies dancing on a figure eight" will also be illustrated.

The organization of this paper reads as follows. In $\S 2$, we formulate the generalized median operator $\mathcal{M}_{p, q}^{\mathrm{wx} / \mathrm{yz}}$ on triangles with two complex parameters $p, q(p q \neq 1)$ and with labels $\mathrm{w}, \mathrm{x}, \mathrm{y}, \mathrm{z} \in \mathbb{Z} / 3 \mathbb{Z}(\mathrm{y} \neq \mathrm{z})$, and illustrate their geometric features on triangles. In $\S 3$, we present how the finite Fourier transformation of triangles improves defects of the original parameters $(p, q)$ so as to introduce $\mathcal{M}^{\mathrm{wx} / \mathrm{yz}}\left[\eta, \eta^{\prime}\right]$ with a new parameter system $\left(\eta, \eta^{\prime}\right) \in \mathbb{C}^{2}$. In particular, $\mathcal{M}^{\mathrm{wx} / \mathrm{yz}}\left[\eta, \eta^{\prime}\right]$ turns out to be expressed as the generalized cevian operator $\mathcal{S}\left[\eta_{0}, \eta_{1}\right]$ studied in [8] with suitable change of variables $\left(\eta, \eta^{\prime}\right) \rightarrow\left(\eta_{0}, \eta_{1}\right)$ (Corollary 3.8). In $\S 4$, we provide a set of symmetric identities among those operators $\mathcal{M}^{\mathrm{wx} / \mathrm{yz}}\left[\eta, \eta^{\prime}\right]$ with variations of labels wx/yz and of parameters $\left(\eta, \eta^{\prime}\right)$, and conclude the prescribed primary goal of the first part of this paper. A short section $\S 5$ is then inserted to introduce the space of triangle shapes (moduli space of similarity classes) from the viewpoint of finite Fourier transformation and Hajja's shape function. We also discuss relationship between Hajja's median operator $\mathcal{H}_{s}$ and a binary Ceva operator $\mathcal{C}_{s}$ of Griffiths, Bényi-Ćurgus type from our viewpoint in complex parameter $s \in \mathbb{C}$. The final section $\S 6$ is devoted to studying tracing orbits of three bodies and present their primary characterization in the form $\mathcal{S}\left[\eta(t), \eta^{\prime}(t)\right]\left(\Delta_{0}\right)$ with certain continuous periodic functions $\eta(t), \eta^{\prime}(t): \mathbb{R} / \mathbb{Z} \rightarrow \mathbb{C}$. We illustrate some examples of area preserving triangle motions and of figure eight orbits. The latter example will be generalized to 3-braiding motions on Lissajous curves in a separate article [5].

\section{Generalized median operators}

Throughout this paper, we use the notations: $i:=\sqrt{-1}, \rho:=e^{2 \pi i / 6}, \omega:=e^{2 \pi i / 3}$.

We consider any triangle lies on the complex plane $\mathbb{C}$ and identify it with the multiset of vertices $\left\{a_{0}, a_{1}, a_{2}\right\}$ on $\mathbb{C}$. It is useful to say that a vector $\Delta=\left(a_{0}, a_{1}, a_{2}\right) \in \mathbb{C}^{3}$ is a triangle triple representing the triangle $\left\{a_{0}, a_{1}, a_{2}\right\}$. A triangle triple $\Delta=\left(a_{0}, a_{1}, a_{2}\right) \in \mathbb{C}^{3}$ will sometimes be written as $\Delta=\left(a_{\mathrm{x}}\right)_{\mathrm{x} \in \mathbb{Z} / 3 \mathbb{Z}}$ after the index set $\{0,1,2\}$ for coordinates being naturally identified with $\mathbb{Z} / 3 \mathbb{Z}$, the ring of integers modulo 3 . by

In [8], for $p, q \in \mathbb{C}$ with $p q \neq 1$, we introduced an operation $\mathcal{S}_{p, q}$ on the triangle triples defined

$$
\mathcal{S}_{p, q}\left(a_{0}, a_{1}, a_{2}\right)=\left(a_{0}^{\prime}, a_{1}^{\prime}, a_{2}^{\prime}\right): \begin{cases}a_{0}^{\prime}= & \alpha_{p, q} a_{0}+\beta_{p, q} a_{1}+\gamma_{p, q} a_{2} ; \\ a_{1}^{\prime}= & \alpha_{p, q} a_{1}+\beta_{p, q} a_{2}+\gamma_{p, q} a_{0} \\ a_{2}^{\prime}= & \alpha_{p, q} a_{2}+\beta_{p, q} a_{0}+\gamma_{p, q} a_{1},\end{cases}
$$

where,

$$
\alpha_{p, q}=\frac{p(1-q)}{1-p q}, \beta_{p, q}=\frac{q(1-p)}{1-p q}, \gamma_{p, q}=\frac{(1-p)(1-q)}{1-p q} .
$$

When $p, q$ are real numbers, $\mathcal{S}_{p, q}(\Delta)$ can be obtained from intersection points of certain two cevian triples of $\Delta$ as introduced in [7]. For convenience, we shall call $\mathcal{S}_{p, q}$ a generalized cevian 
operator on triangles also for complex parameters $p, q$. Since $\alpha_{p, q}+\beta_{p, q}+\gamma_{p, q}=1$, it is easy to see that the centroids of $\Delta=\left(a_{0}, a_{1}, a_{2}\right)$ and of $\Delta^{\prime}:=\mathcal{S}_{p, q}(\Delta)=\left(a_{0}^{\prime}, a_{1}^{\prime}, a_{2}^{\prime}\right)$ coincide and that

$$
\sum_{k \in \mathbb{Z} / 3 \mathbb{Z}} \overrightarrow{a_{\mathrm{w}+k} a_{\mathbf{x}+k}^{\prime}}=\mathbf{0}
$$

for any choice of $\mathrm{w}, \mathrm{x} \in \mathbb{Z} / 3 \mathbb{Z}$. This determines, for each $(\mathrm{y}, \mathrm{z}) \in(\mathbb{Z} / 3 \mathbb{Z})^{2}$ with $\mathrm{y} \neq \mathrm{z}$, a unique triangle triple $\Delta^{\prime \prime}=\left(a_{0}^{\prime \prime}, a_{1}^{\prime \prime}, a_{2}^{\prime \prime}\right)$ by the conditions:

$$
\Delta^{\prime \prime} \text { shares the centroid with } \Delta, \Delta^{\prime} \text {, }
$$$$
\text { in other words, the three triangles } \Delta, \Delta^{\prime} \text { and } \Delta^{\prime \prime} \text { are concentroid; }
$$

$$
\overrightarrow{a_{\mathrm{w}+k} a_{\mathrm{x}+k}^{\prime}}=\overrightarrow{a_{\mathrm{y}+k}^{\prime \prime} a_{\mathrm{z}+k}^{\prime \prime}} \quad(k \in \mathbb{Z} / 3 \mathbb{Z}) .
$$

Definition $2.5((p, q)$-median triangle). Let $p, q \in \mathbb{C}$ with $p q \neq 1$, and $\mathrm{w}, \mathrm{x}, \mathrm{y}, \mathrm{z} \in \mathbb{Z} / 3 \mathbb{Z}$ with $\mathrm{y} \neq \mathrm{z}$. Given a triangle triple $\Delta=\left(a_{0}, a_{1}, a_{2}\right)$ with $\Delta^{\prime}=\mathcal{S}_{p, q}(\Delta)=\left(a_{0}^{\prime}, a_{1}^{\prime}, a_{2}^{\prime}\right)$, we define the triangle triple

$$
\mathcal{M}_{p, q}^{\mathrm{wx} / \mathrm{yz}}(\Delta):=\Delta^{\prime \prime}
$$

where $\Delta^{\prime \prime}=\left(a_{0}^{\prime \prime}, a_{1}^{\prime \prime}, a_{2}^{\prime \prime}\right)$ is determined by the condition 2.3$)-2.4$. We shall call $\mathcal{M}_{p, q}^{\mathrm{wx} / \mathrm{yz}}$ a generalized median operator on triangles.

Before focusing on specific examples, let us here illustrate actions of $\mathcal{M}_{p, q}^{\mathrm{wx} / \mathrm{yz}}$ in a generic sample case: Let $\Delta=\left(a_{0}, a_{1}, a_{2}\right)$ be a triangle $\left(0,1, \frac{7+8 i}{10}\right)$ and let complex parameter $(p, q)$ be set as $\left(\frac{4}{5}, \frac{2+4 i}{3}\right)$. Then, the generalized cevian operator $\mathcal{S}_{p, q}$ maps $\Delta$ to $\Delta^{\prime}=\left(a_{0}^{\prime}, a_{1}^{\prime}, a_{2}^{\prime}\right)=$ $\left(\frac{93}{3050}+\frac{542}{1525} i, \frac{201}{305}-\frac{46}{305} i, \frac{1541}{1525}+\frac{908}{1525} i\right)$. One can form a triangle $\Delta^{\prime \prime}=\left(a_{0}^{\prime \prime}, a_{1}^{\prime \prime}, a_{2}^{\prime \prime}\right)$ formed by the sides parallel to the three vectors $\overrightarrow{a_{0} a_{0}^{\prime}}, \overrightarrow{a_{1} a_{1}^{\prime}}, \overrightarrow{a_{2} a_{2}^{\prime}}$. Here arise six-fold ways to label the vertices $a_{0}^{\prime \prime}, a_{1}^{\prime \prime}, a_{2}^{\prime \prime}$ depending on choices of pairs $(\mathrm{y}, \mathrm{z})$ with $\mathrm{y}, \mathrm{z} \in\{0,1,2\}, \mathrm{y} \neq \mathrm{z}$ so that $\overrightarrow{a_{0} a_{0}^{\prime}}=\overrightarrow{a_{\mathrm{y}}^{\prime \prime} a_{\mathrm{z}}^{\prime \prime}}$. Every such a choice yields the generalized median triangle $\mathcal{M}_{p, q}^{00 / \mathrm{yz}}(\Delta)$. The following picture (Figure 1) illustrates $\mathcal{M}_{p, q}^{00 / 01}(\Delta)$, one of those six choices, such that $\overrightarrow{a_{0} a_{0}^{\prime}}=\overrightarrow{a_{0}^{\prime \prime} a_{1}^{\prime \prime}}$. We also note that $\mathcal{M}_{p, q}^{00 / 01}=\mathcal{M}_{p, q}^{11 / 12}=\mathcal{M}_{p, q}^{22 / 20}$ by definition.

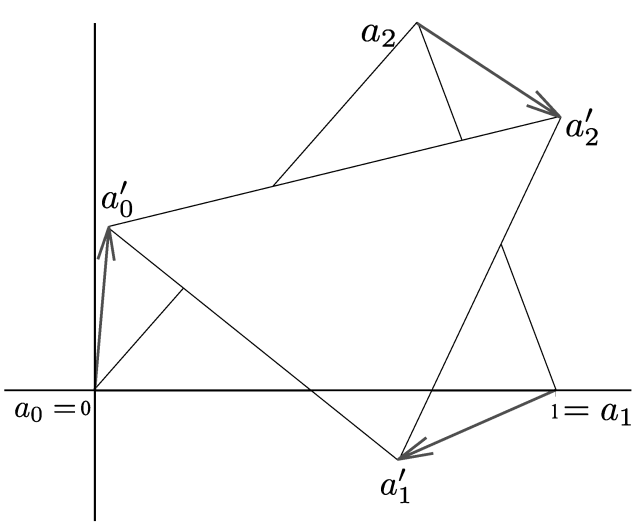

$$
\Delta=\left(0,1, \frac{7+8 i}{10}\right) \text { and } \Delta^{\prime}=\mathcal{S}_{\frac{4}{5}}, \frac{2+4 i}{3}(\Delta)
$$

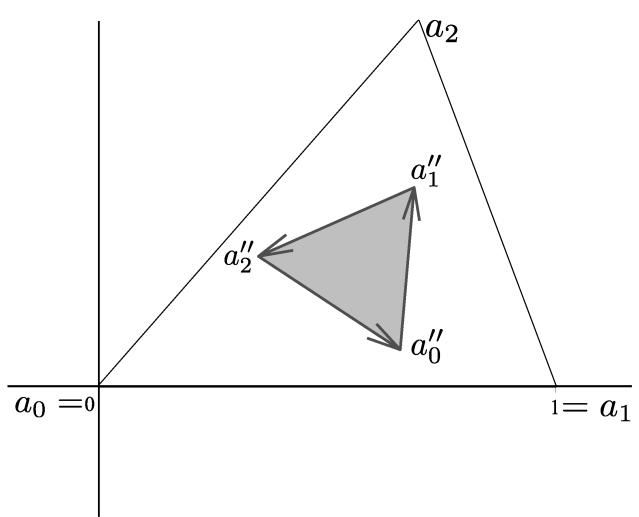

$\Delta$ and $\Delta^{\prime \prime}=\mathcal{M}_{\frac{4}{5}, \frac{2+4 i}{3}}^{00 / 01}(\Delta)$

FiguRE 1. Illustration of $\mathcal{S}_{p, q}$ and $\mathcal{M}_{p, q}^{00 / 01}$ 
There is another set of six-fold ways to form $\Delta^{\prime \prime}=\left(a_{0}^{\prime \prime}, a_{1}^{\prime \prime}, a_{2}^{\prime \prime}\right)$ whose sides are taken to be parallel to $\overrightarrow{a_{0} a_{1}^{\prime}}, \overrightarrow{a_{1} a_{2}^{\prime}}, \overrightarrow{a_{2} a_{0}^{\prime}}$ in total. The following picture (Figure 2 ) shows one of those cases $\mathcal{M}_{p, q}^{01 / 01}(\Delta)$ where $a_{0}^{\prime \prime}, a_{1}^{\prime \prime}, a_{2}^{\prime \prime}$ are labeled to satisfy $\overrightarrow{a_{0} a_{1}^{\prime}}=\overrightarrow{a_{0}^{\prime \prime} a_{1}^{\prime \prime}}$. We also note that $\mathcal{M}_{p, q}^{01 / 01}=$ $\mathcal{M}_{p, q}^{12 / 12}=\mathcal{M}_{p, q}^{20 / 20}$ by definition.

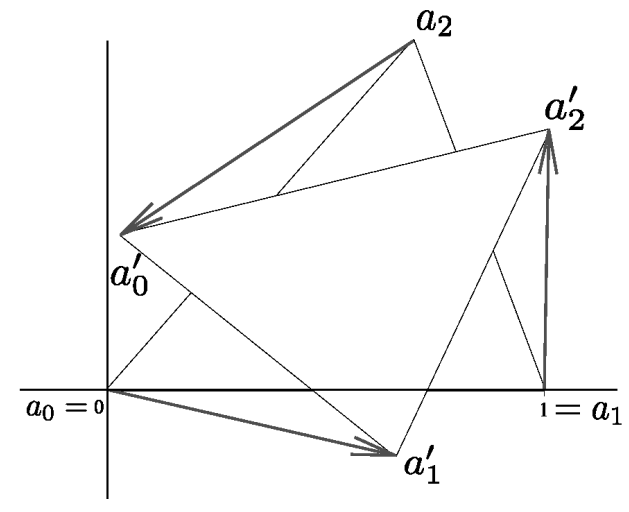

$$
\Delta=\left(0,1, \frac{7+8 i}{10}\right) \text { and } \Delta^{\prime}=\mathcal{S}_{\frac{4}{5}}, \frac{2+4 i}{3}(\Delta)
$$

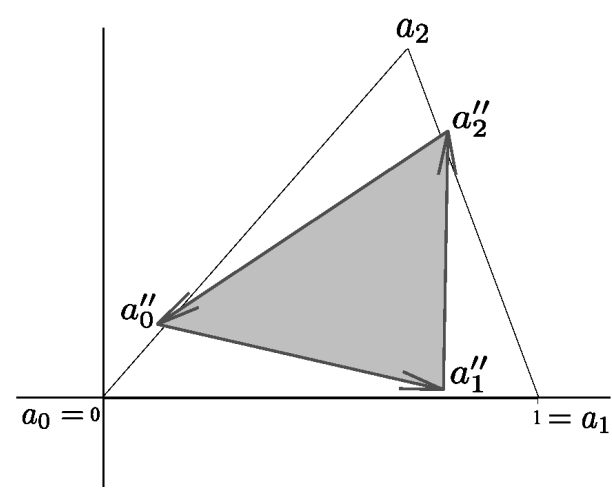

$\Delta$ and $\Delta^{\prime \prime}=\mathcal{M}_{\frac{4}{5}, \frac{2+4 i}{3}}^{01 / 01}(\Delta)$

FiguRE 2. Illustration of $\mathcal{S}_{p, q}$ and $\mathcal{M}_{p, q}^{01 / 01}$

It remains to take $\Delta^{\prime \prime}=\left(a_{0}^{\prime \prime}, a_{1}^{\prime \prime}, a_{2}^{\prime \prime}\right)$ formed by three sides parallel to $\overrightarrow{a_{0} a_{2}^{\prime}}, \overrightarrow{a_{1} a_{0}^{\prime}}, \overrightarrow{a_{2} a_{1}^{\prime}}$. Again we have six-fold ways to label the vertices of $\Delta^{\prime \prime}$ subject to $\overrightarrow{a_{0} a_{2}^{\prime}}=\overrightarrow{a_{\mathrm{y}}^{\prime \prime} a_{\mathrm{z}}^{\prime \prime}}(\mathrm{y}, \mathrm{z} \in\{0,1,2\}$, $\mathrm{y} \neq \mathbf{z}$ ). The following picture (Figure 3) illustrates the case $\mathrm{y}=0, \mathbf{z}=1$. We also note that $\mathcal{M}_{p, q}^{02 / 01}=\mathcal{M}_{p, q}^{10 / 12}=\mathcal{M}_{p, q}^{21 / 20}$ by definition.

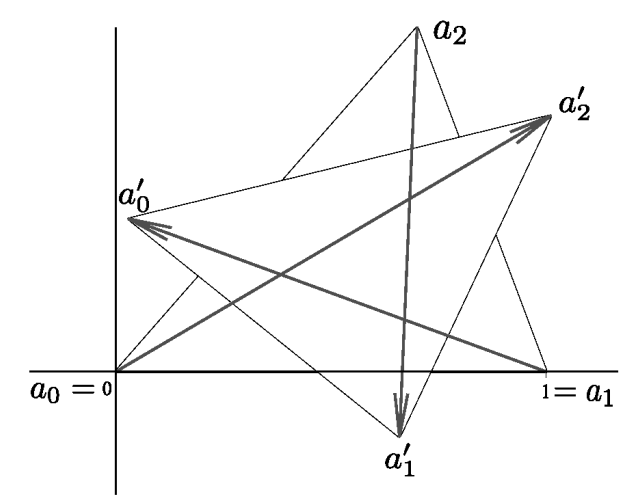

$$
\Delta=\left(0,1, \frac{7+8 i}{10}\right) \text { and } \Delta^{\prime}=\mathcal{S}_{\frac{4}{5}}, \frac{2+4 i}{3}(\Delta)
$$

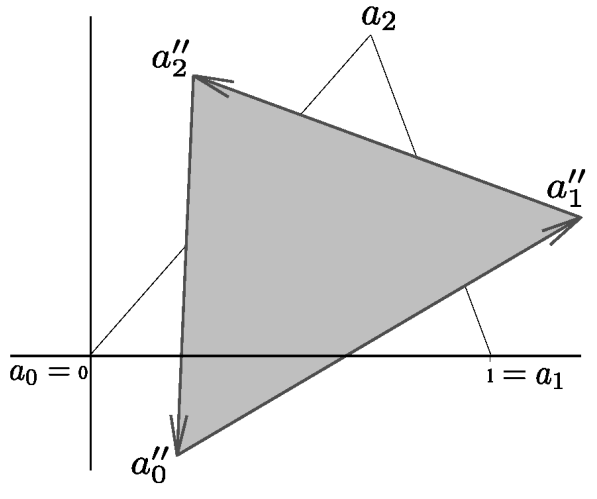

$\Delta$ and $\Delta^{\prime \prime}=\mathcal{M}_{\frac{4}{5}, \frac{2+4 i}{3}}^{02 / 01}(\Delta)$

FiguRE 3. Illustration of $\mathcal{S}_{p, q}$ and $\mathcal{M}_{p, q}^{02 / 01}$

As shown in the above description, we generally have $18(=3 \times 6)$-fold ways to define $\Delta^{\prime \prime}=$ $\mathcal{M}_{p, q}^{\mathrm{wx} / \mathrm{yz}}(\Delta)$ whose sides are composed of three disjoint bridges between the vertices of $\Delta$ and of $\mathcal{S}_{p, q}(\Delta)$. In $\S 4$, we will discuss precise relations among those 18 -fold ways at the operator level. 
In the next two examples, we focus on some specific cases which connect $\mathcal{M}_{p, q}^{\mathrm{wx} / \mathrm{yz}}(\Delta)$ to its historical origins.

Example 2.6 (Prototype). Let $\triangle A B C$ be a triangle represented by a triple $\Delta=(a, b, c) \in \mathbb{C}^{3}$. Let us illustrate the classical case in Introduction in our terminology: As noted in [7, Example 1.2], the midpoint triangle $\mathcal{S}(\Delta)=\Delta A^{\prime} B^{\prime} C^{\prime}$ is given by $\mathcal{S}_{0, \frac{1}{2}}(\Delta)$. The median triangle $\mathcal{M}(\Delta)=$ $\Delta A^{\prime \prime} B^{\prime \prime} C^{\prime \prime}$ labeled by the condition $\overrightarrow{A A^{\prime}}=\overrightarrow{A^{\prime \prime} B^{\prime \prime}}, \overrightarrow{B B^{\prime}}=\overrightarrow{B^{\prime \prime} C^{\prime \prime}}, \overrightarrow{C C^{\prime}}=\overrightarrow{C^{\prime \prime} A^{\prime \prime}}$ is then given by $\mathcal{M}_{0, \frac{1}{2}}^{00 / 01}(\Delta)$.

Example 2.7. In [4], M.Hajja discusses three types of triangles called the $s$-medial, the $s$-Routh, and the $s$-median triangles with a real parameter $s \in \mathbb{R}$. The $(p, q)$-median triangle introduced above generalizes Hajja's $s$-median triangle. Start with a triangle $\triangle A B C$ represented by a positive triangle triple $\Delta=(a, b, c)$ satisfying $\operatorname{Im}\left(\frac{a-b}{c-b}\right)>0$. Form first $\Delta^{\prime}=\left(a^{\prime}, b^{\prime}, c^{\prime}\right)$ to be $\mathcal{S}_{0,1-s}(\Delta)$ (called the $s$-medial triangle of $\left.\Delta\right)$, the triangle whose vertices are $(s: 1-s)$-division points of the edges of $\Delta$. The $s$-median triangle of $\Delta$, written $\mathcal{H}_{s}(\Delta)$ is, by definition, a triangle $\left\{a^{\prime \prime}, b^{\prime \prime}, c^{\prime \prime}\right\}$ such that $\overrightarrow{a a^{\prime}}=\overrightarrow{b^{\prime \prime} c^{\prime \prime}}, \overrightarrow{b b^{\prime}}=\overrightarrow{c^{\prime \prime} a^{\prime \prime}}$, and $\overrightarrow{c c^{\prime}}=\overrightarrow{a^{\prime \prime} b^{\prime \prime}}$. Without loss of generality, we may assume $\mathcal{H}_{s}(\Delta)$ and $\Delta$ are concentroid, i.e. $a+b+c=a^{\prime \prime}+b^{\prime \prime}+c^{\prime \prime}$ so that $\mathcal{H}_{s}(\Delta)$ is uniquely determined from $\Delta$. In our above definition, we find $\mathcal{H}_{s}(\Delta)$ to be $\mathcal{M}_{0,1-s}^{00 / 12}(\Delta)$.

\section{Fourier parameters}

The collection of operators $\mathbb{S}^{\prime}:=\left\{\mathcal{S}_{p, q} \mid(p, q) \in \mathbb{C}^{2}, p q \neq 1\right\}$ is incomplete in the sense that the composition $\mathcal{S}_{p_{1}, q_{1}} \mathcal{S}_{p_{2}, q_{2}}$ may not always be of the form of an $\mathcal{S}_{p, q} \in \mathbb{S}^{\prime}$. The lesson found in our previous work [8] to remedy this defect is to introduce the Fourier transforms $\Psi(\Delta)$ for triangles $\Delta=(a, b, c)$ by

$$
\Psi(\Delta)=\left(\begin{array}{l}
\psi_{0}(\Delta) \\
\psi_{1}(\Delta) \\
\psi_{2}(\Delta)
\end{array}\right)=\frac{1}{3}\left(\begin{array}{c}
a+b+c \\
a+b \omega^{2}+c \omega \\
a+b \omega+c \omega^{2}
\end{array}\right)
$$

and to replace the parameter $(p, q) \in \mathbb{C}^{2}(p q \neq 1)$ by a new parameter $\left(\eta, \eta^{\prime}\right) \in \mathbb{C}^{2}$ defined by

$$
\eta:=\frac{p-q}{1-p q}+\frac{(p-1)(2 q-1)}{1-p q} \omega, \quad \eta^{\prime}:=\frac{p-q}{1-p q}+\frac{(p-1)(2 q-1)}{1-p q} \omega^{2} .
$$

Indeed, with these parameters, the operator $\mathcal{S}_{p, q}$ is diagonalized as mapping $\Delta$ to $\Delta^{\prime}$ in the form

$$
\psi_{0}\left(\Delta^{\prime}\right)=\psi_{0}(\Delta), \quad \psi_{1}\left(\Delta^{\prime}\right)=\eta^{\prime} \cdot \psi_{1}(\Delta), \quad \psi_{2}\left(\Delta^{\prime}\right)=\eta \cdot \psi_{2}(\Delta) .
$$

It turns out that the collection $\mathbb{S}^{\prime}:=\left\{\mathcal{S}_{p, q} \mid(p, q) \in \mathbb{C}^{2}, p q \neq 1\right\}$ extends to a more complete family

$$
\mathbb{S}:=\left\{\mathcal{S}\left[\eta, \eta^{\prime}\right] \mid\left(\eta, \eta^{\prime}\right) \in \mathbb{C}^{2}\right\}
$$

by identifying $\mathcal{S}_{p, q}=\mathcal{S}\left[\eta, \eta^{\prime}\right]$ for $p q \neq 1$ so that the composition law $\mathcal{S}\left[\eta_{1}, \eta_{1}^{\prime}\right] \mathcal{S}\left[\eta_{2}, \eta_{2}^{\prime}\right]=$ $\mathcal{S}\left[\eta_{1} \eta_{2}, \eta_{1}^{\prime} \eta_{2}^{\prime}\right]$ provides a natural multiplicative monoid structure on $\mathbb{S}$.

Now, regarding triangle triples as column vectors in $\mathbb{C}^{3}$, we easily see that the operations $\mathcal{S}_{p, q}$ and $\mathcal{S}\left[\eta, \eta^{\prime}\right]$ naturally determine linear transformations $\left(3\right.$ by 3 matrices in $M_{3}(\mathbb{C})$ ) acting on $\mathbb{C}^{3}$ on the left. Below, we shall identify those operators as their matrix representatives in $M_{3}(\mathbb{C})$. Let

$$
\mathrm{I}:=\left(\begin{array}{ccc}
1 & 0 & 0 \\
0 & 1 & 0 \\
0 & 0 & 1
\end{array}\right), \quad \mathrm{J}:=\left(\begin{array}{ccc}
0 & 1 & 0 \\
0 & 0 & 1 \\
1 & 0 & 0
\end{array}\right), \quad W:=\left(\begin{array}{ccc}
1 & 1 & 1 \\
1 & \omega & \omega^{2} \\
1 & \omega^{2} & \omega
\end{array}\right)
$$


Note that the above Fourier transform (3.1) may be written in the matrix multiplication form: $\Psi\left(\left(\begin{array}{l}a \\ b \\ c\end{array}\right)\right)=W^{-1}\left(\begin{array}{l}a \\ b \\ c\end{array}\right)$. The following proposition summarizes basic properties for $\mathcal{S}\left[\eta, \eta^{\prime}\right] \in \mathbb{S}$ :

Proposition 3.5 ([8]). Notations being as above, we have:

(i) $\mathbb{S}=\left\{\alpha \mathbf{l}+\beta \mathbf{J}+\gamma \mathrm{J}^{2} \mid \alpha+\beta+\gamma=1\right\} \subset M_{3}(\mathbb{C})$.

(ii) $\mathcal{S}\left[\eta, \eta^{\prime}\right]=W \cdot \operatorname{diag}\left(1, \eta^{\prime}, \eta\right) \cdot W^{-1}$

$$
=\frac{1}{3}\left(1+\eta+\eta^{\prime}\right) \boldsymbol{I}+\frac{1}{3}\left(1+\eta \omega+\eta^{\prime} \omega^{2}\right) \mathrm{J}+\frac{1}{3}\left(1+\eta \omega^{2}+\eta^{\prime} \omega\right) \mathrm{J}^{2} \quad\left(\eta, \eta^{\prime} \in \mathbb{C}\right) .
$$

Let us turn to generalized median operators. We first extend $\mathcal{M}_{p, q}^{\mathrm{wx} / \mathrm{yz}}(p q \neq 1)$ to the new parameters $\left(\eta, \eta^{\prime}\right) \in \mathbb{C}^{2}$. Below, we understand the number $\omega^{\mathrm{x}}$ and the matrix $\mathrm{J}^{\mathrm{x}}$ in the obvious sense for each $\mathrm{x} \in \mathbb{Z} / 3 \mathbb{Z}$.

Definition $3.6\left(\left(\eta, \eta^{\prime}\right)\right.$-median triangles). Let $\eta, \eta^{\prime} \in \mathbb{C}$, and let $\mathrm{w}, \mathrm{x}, \mathrm{y}, \mathrm{z} \in \mathbb{Z} / 3 \mathbb{Z}$ with y $\neq \mathrm{z}$. Given a triangle triple $\Delta=\left(a_{0}, a_{1}, a_{2}\right)$ with $\Delta^{\prime}=\mathcal{S}\left[\eta, \eta^{\prime}\right](\Delta)=\left(a_{0}^{\prime}, a_{1}^{\prime}, a_{2}^{\prime}\right)$, we define the triangle triple

$$
\mathcal{M}^{\mathrm{wx} / \mathrm{yz}}\left[\eta, \eta^{\prime}\right](\Delta):=\Delta^{\prime \prime}
$$

where $\Delta^{\prime \prime}=\left(a_{0}^{\prime \prime}, a_{1}^{\prime \prime}, a_{2}^{\prime \prime}\right)$ is determined by the condition $(2.3)-2.4$.

It is not difficult to see that $\mathcal{M}^{\mathrm{wx} / \mathrm{yz}}\left[\eta, \eta^{\prime}\right] \in \mathbb{S}$. In fact, we have the following explicit formula:

Proposition 3.7. Given $\eta, \eta^{\prime} \in \mathbb{C}$ and $\mathrm{w}, \mathrm{x}, \mathrm{y}, \mathrm{z} \in \mathbb{Z} / 3 \mathbb{Z}$ with $\mathrm{y} \neq \mathrm{z}$, we have

$$
\left(\mathrm{J}^{\mathrm{z}}-\mathrm{J}^{\mathrm{y}}\right) \mathcal{M}^{\mathrm{wx} / \mathrm{yz}}\left[\eta, \eta^{\prime}\right]=\mathrm{J}^{\mathrm{x}} \mathcal{S}\left[\eta, \eta^{\prime}\right]-\mathrm{J}^{\mathrm{w}} .
$$

Proof. Let $\Delta=\left(a_{0}, a_{1}, a_{2}\right)$ be a triangle triple, and write $\Delta^{\prime}=\mathcal{S}\left[\eta, \eta^{\prime}\right](\Delta)=\left(a_{0}^{\prime}, a_{1}^{\prime}, a_{2}^{\prime}\right)$ and $\Delta^{\prime \prime}=\mathcal{M}^{\mathrm{wx} / \mathrm{yz}}\left[\eta, \eta^{\prime}\right](\Delta)=\left(a_{0}^{\prime \prime}, a_{1}^{\prime \prime}, a_{2}^{\prime \prime}\right)$. The assertion essentially amounts to seeing the identity

$$
\left(\mathrm{J}^{\mathrm{z}}-\mathrm{J}^{\mathrm{y}}\right)\left(\Delta^{\prime \prime}\right)=\mathrm{J}^{\mathrm{x}}\left(\Delta^{\prime}\right)-\mathrm{J}^{\mathrm{w}}(\Delta) .
$$

Observe that the 1st component of $\mathrm{J}^{\mathrm{x}}\left(\Delta^{\prime}\right)-\mathrm{J}^{\mathrm{w}}(\Delta)$ is $\overrightarrow{a_{\mathrm{w}} a_{\mathrm{x}}^{\prime}}$, and that the 1st component of $\mathrm{J}^{\mathrm{z}}\left(\Delta^{\prime \prime}\right)-\mathrm{J}^{\mathrm{y}}\left(\Delta^{\prime \prime}\right)$ is $\overrightarrow{a_{\mathrm{y}}^{\prime \prime} a_{\mathrm{z}}^{\prime \prime}}$. They coincide with each other by definition. Similarly, one can see the coincidence of their 2nd and 3rd components, as they are the 1st components of the above after $\Delta$ replaced by $\mathrm{J} \Delta, \mathrm{J}^{2} \Delta$. One can extend the identity also for degenerate triangle triples by easy argument of continuity, and hence conclude the matrix identity as asserted.

Although the factor $\left(\mathrm{J}^{\mathrm{z}}-\mathrm{J} \mathrm{y}\right)$ in LHS of the above Proposition 3.7 is not an invertible matrix, the concentroid condition $(2.3)$ determines $\mathcal{M}^{\mathrm{wx} / \mathrm{yz}}\left[\eta, \eta^{\prime}\right]$ in $\mathbb{S}$ as seen in the following corollary. In fact, the generalized median operator $\mathcal{M}^{\mathrm{wx} / \mathrm{yz}}\left[\eta, \eta^{\prime}\right]$ turns out to be reduced to a generalized cevian operator $\mathcal{S}\left[\eta_{0}, \eta_{1}\right]$ after a simple change of parameters:

Corollary 3.8. Notations being as in Proposition 3.7, we have

$$
\mathcal{M}^{\mathrm{wx} / \mathrm{yz}}\left[\eta, \eta^{\prime}\right]=\mathcal{S}\left[\eta_{0}, \eta_{1}\right]
$$

where

$$
\eta_{0}=\frac{\eta \omega^{-\mathrm{x}}-\omega^{-\mathrm{w}}}{\omega^{-\mathrm{z}}-\omega^{-\mathrm{y}}}, \quad \eta_{1}=\frac{\eta^{\prime} \omega^{\mathrm{x}}-\omega^{\mathrm{w}}}{\omega^{\mathrm{z}}-\omega^{\mathrm{y}}}
$$

Proof. Let $N=\frac{1}{3}\left(\mathrm{I}+\mathrm{J}+\mathrm{J}^{2}\right)$ (i.e., the matrix with all entries $\left.\frac{1}{3}\right)$ so that $N(\Delta)=(g, g, g)$ for every triangle $\Delta=(a, b, c)$ with centroid $g=\frac{1}{3}(a+b+c)$. Since $M=\mathcal{M}^{\mathrm{wx} / \mathrm{yz}}\left[\eta, \eta^{\prime}\right]$ preserves centroids of triangles, we have $N M(\Delta)=N(\Delta)$ for all $\Delta$, hence have the identity $N M=N$. It follows then from Proposition 3.7 that $(*):\left(\mathrm{J}^{\mathrm{z}}-\mathrm{J}^{\mathrm{y}}+N\right) M=\mathrm{J}^{\mathrm{x}} \mathcal{S}\left[\eta, \eta^{\prime}\right]-\mathrm{J}^{\mathrm{w}}+N$. Since the 
matrix $\left(\mathrm{J}^{\mathrm{z}}-\mathrm{J}^{\mathrm{y}}+N\right) \in \mathbb{S}$ is invertible, the identity $(*)$ determines $M$ which itself lies in $\mathbb{S}$ by Proposition 3.5 (ii) and gives rise to

$$
\left[\begin{array}{lll}
0 & & \\
& \omega^{\mathrm{z}}-\omega^{\mathrm{y}} & \\
& & \omega^{-\mathrm{z}}-\omega^{-\mathrm{y}}
\end{array}\right]\left[\begin{array}{lll}
1 & & \\
& \eta_{1} & \\
& & \eta_{0}
\end{array}\right]=\left[\begin{array}{lll}
1 & & \\
& \omega^{\mathrm{x}} & \\
& & \omega^{-\mathrm{x}}
\end{array}\right]\left[\begin{array}{lll}
1 & & \\
& \eta^{\prime} & \\
& & \eta
\end{array}\right]-\left[\begin{array}{lll}
1 & & \\
& \omega^{\mathrm{w}} & \\
& & \omega^{-\mathrm{w}}
\end{array}\right]
$$

after conjugation by $W$. This settles the asserted formula on $\left(\eta_{0}, \eta_{1}\right)$.

\section{Reduction of $\mathbf{1 8}$-fold ways of $\mathcal{M}^{\text {wx } / \mathrm{yz}}$}

The upper label $\mathrm{wx} / \mathrm{yz}$ for a generalzed median operator $\mathcal{M}^{\mathrm{wx} / \mathrm{yz}}\left[\eta, \eta^{\prime}\right]$ is to be given from the collection of $(\mathrm{w}, \mathrm{x}, \mathrm{y}, \mathrm{z}) \in(\mathbb{Z} / 3 \mathbb{Z})^{4}$ with $\mathrm{y} \neq \mathrm{z}$.

Since the condition (2.4) is stable under simultaneous shifts of labels in wx/yz, we have the identity $\mathcal{M}^{\mathrm{wx} / \mathrm{yz}}\left[\eta, \eta^{\prime}\right]=\mathcal{M}^{\mathrm{w}+1, \mathrm{x}+1 / \mathrm{y}+1, \mathrm{z}+1}\left[\eta, \eta^{\prime}\right]$ which will be listed below in 4.2 . As a consequence, there are 18 different ways of labels up to the shifts in $\mathbb{Z} / 3 \mathbb{Z}$. However, there are many other identities which co-relate generalized cevian and median operators as shown in the following list (4.1)- 4.8 ).

$$
\begin{aligned}
& \mathcal{S}\left[\eta, \eta^{\prime}\right]=\mathcal{S}\left[\eta \omega, \eta^{\prime} \omega^{-1}\right] \cdot \mathrm{J}=\mathrm{J} \cdot \mathcal{S}\left[\eta \omega, \eta^{\prime} \omega^{-1}\right] \\
& \mathcal{M}^{\mathrm{wx} / \mathrm{yz}}\left[\eta, \eta^{\prime}\right]=\mathcal{M}^{\mathrm{w}+1, \mathrm{x}+1 / \mathrm{y}+1, \mathrm{z}+1}\left[\eta, \eta^{\prime}\right] \\
& \mathcal{M}^{\mathrm{wx} / \mathrm{yz}}\left[\eta, \eta^{\prime}\right] \cdot \mathrm{J}=\mathcal{M}^{\mathrm{w}+1, \mathrm{x}+1 / \mathrm{yz}}\left[\eta, \eta^{\prime}\right] \\
& \mathcal{M}^{\mathrm{wx} / \mathrm{yz}}\left[\eta, \eta^{\prime}\right] \cdot \mathrm{J}^{2}=\mathcal{M}^{\mathrm{wx} / \mathrm{y}+1, \mathrm{z}+1}\left[\eta, \eta^{\prime}\right] \\
& \mathcal{M}^{\mathrm{wx} / \mathrm{yz}}\left[\eta, \eta^{\prime}\right]=\mathcal{M}^{\mathrm{w}, \mathrm{x}+1 / \mathrm{yz}}\left[\eta \omega, \eta^{\prime} \omega^{-1}\right]=\mathcal{M}^{\mathrm{w}, \mathrm{x}-1 / \mathrm{yz}}\left[\eta \omega^{-1}, \eta^{\prime} \omega\right] \\
& \frac{1}{3}\left(\mathcal{M}^{\mathrm{w} 0 / \mathrm{yz}}\left[\eta, \eta^{\prime}\right]+\mathcal{M}^{\mathrm{w} 1 / \mathrm{yz}}\left[\eta, \eta^{\prime}\right]+\mathcal{M}^{\mathrm{w} 2 / \mathrm{yz}}\left[\eta, \eta^{\prime}\right]\right)=\frac{1}{3} \mathrm{~J}^{\mathrm{w}+\mathrm{y}+\mathrm{z}}+\frac{2}{3} \mathrm{~J}^{\mathrm{w}-\mathrm{y}} \\
& \frac{1}{2}\left(\mathcal{M}^{\mathrm{wx} / \mathrm{yz}}\left[\eta, \eta^{\prime}\right]+\mathcal{M}^{\mathrm{wx} / \mathrm{zy}}\left[\eta, \eta^{\prime}\right]\right)=\mathcal{S}_{\frac{1}{2}, \frac{1}{2}}=\frac{1}{3}\left(\mathrm{I}+\mathrm{J}+\mathrm{J}^{2}\right) \\
& \mathcal{M}^{\mathrm{wx} / \mathrm{yz}}\left[\omega^{\mathrm{x}-\mathrm{w}}+\eta, \omega^{\mathrm{w}-\mathrm{x}}+\eta^{\prime}\right]=\mathrm{J}^{\mathrm{x}} \cdot \mathcal{M}^{00 / \mathrm{yz}}\left[1+\eta, 1+\eta^{\prime}\right] .
\end{aligned}
$$

Proof of (4.1)- (4.8). Proposition 3.5 and Corollary 3.8 enable one to express $\mathcal{S}\left[\eta, \eta^{\prime}\right]$ and $\mathcal{M}^{\mathrm{wx} / \mathrm{yz}}\left[\eta, \eta^{\prime}\right]$ in $\mathbb{S}$ as explicit 3 by 3 matrices for every $\left(\eta, \eta^{\prime}\right) \in \mathbb{C}^{2}$ and for wx/yz. Then the proofs of these identities, once discovered, can be easily verified (say, by using symbolic computer systems).

Interpretation: Let $\Delta$ be a triangle triple and fix a pair of parameters $\left(\eta, \eta^{\prime}\right) \in \mathbb{C}^{2}$. The relation 4.7) tells that $\mathcal{M}^{\mathrm{wx} / \mathrm{yz}}\left[\eta, \eta^{\prime}\right](\Delta)$ and $\mathcal{M}^{\mathrm{wx} / \mathrm{zy}}\left[\eta, \eta^{\prime}\right](\Delta)$ are point-symmetrical about the centroid of $\Delta$. This together with $(4.2)$ implies that $\mathcal{M}^{\mathrm{wx} / \mathrm{y}, \mathrm{y}+1}\left[\eta, \eta^{\prime}\right](\Delta)(\mathrm{w}, \mathrm{x}, \mathrm{y} \in \mathbb{Z} / 3 \mathbb{Z})$ give all possible triangle triples up to point symmetry. Consider, then, effects of (4.3) and (4.4) after remarking that the action of $\mathrm{J}$ on triangle triples changes only labels of vertices. (Note also that every matrix in $\mathbb{S}$ commutes with $J$.) From this we realize that the three median triangles

$$
\mathcal{M}^{00 / 01}\left[\eta, \eta^{\prime}\right](\Delta), \mathcal{M}^{01 / 01}\left[\eta, \eta^{\prime}\right](\Delta), \mathcal{M}^{02 / 01}\left[\eta, \eta^{\prime}\right](\Delta)
$$

provide all possibly different triangles from 18 -fold triples $\mathcal{M}^{\mathrm{wx} / \mathrm{yz}}\left[\eta, \eta^{\prime}\right](\Delta)$ in $(\mathrm{w}, \mathrm{x}, \mathrm{y}, \mathrm{z}) \in$ $(\mathbb{Z} / 3 \mathbb{Z})^{4}$ with $y \neq z$ (up to parallelism, point symmetry and label permutations). Note also that the last three triangles are also dependent by a linear relation $(4.6)$. 
As illustrated in [8, Remark 3.6], the operations $\mathcal{S}_{p, q}$ have closer geometrical interpretation on triangles with respect to the original parameters $p, q \in \mathbb{C}(p q \neq 1)$. In the rest of this section, we shall translate the above results for $\mathcal{S}\left[\eta, \eta^{\prime}\right], \mathcal{M}^{\mathrm{wx} / \mathrm{yz}}\left[\eta, \eta^{\prime}\right]$ into the context of $\mathcal{S}_{p, q}, \mathcal{M}_{p, q}^{\mathrm{wx} / \mathrm{yz}}$.

Noting that the transformation $(3.2)$ is birational with

$$
p=\frac{1+\eta+\eta^{\prime}}{2-\omega \eta-\omega^{2} \eta^{\prime}}, \quad q=\frac{1+\omega \eta+\omega^{2} \eta^{\prime}}{2-\eta-\eta^{\prime}}
$$

(cf. [8, Prop. 5.13]), we translate Corollary 3.8 in the form

$$
\mathcal{M}_{p, q}^{\mathrm{wx} / \mathrm{yz}}=\mathcal{S}_{p_{1}, q_{1}}
$$

with $p_{1}, q_{1}$ suitable rational functions in $p, q$ and vice versa. The following table shows some samples chosen from 18 types of labels, where

$$
\Delta^{\prime \prime}=\left(a_{0}^{\prime \prime}, a_{1}^{\prime \prime}, a_{2}^{\prime \prime}\right)=\mathcal{M}_{p, q}^{\mathrm{wx} / \mathrm{yz}}(\Delta)=\mathcal{S}_{p_{1}, q_{1}}(\Delta)
$$

for $\Delta=\left(a_{0}, a_{1}, a_{2}\right)$ and $\Delta^{\prime}=\left(a_{0}^{\prime}, a_{1}^{\prime}, a_{2}^{\prime}\right)=\mathcal{S}_{p, q}(\Delta)$ :

TABLE $1 . \mathcal{M}_{p, q}^{\mathrm{wx} / \mathrm{yz}}(\Delta)=\mathcal{S}_{p_{1}, q_{1}}(\Delta)$

\begin{tabular}{|l|l|c|c|}
\hline wx $/ \mathrm{yz}$ & $\overrightarrow{a_{\mathrm{w}} a_{\mathrm{x}}^{\prime}}=\overrightarrow{a_{\mathrm{y}}^{\prime \prime} a_{\mathrm{z}}^{\prime \prime}}$ & {$\left[p_{1}, q_{1}\right]$} & {$[p, q]$} \\
\hline $00 / 01$ & $\overrightarrow{a_{0} a_{0}^{\prime}}=\overrightarrow{a_{0}^{\prime \prime} a_{1}^{\prime \prime}}$ & {$\left[\frac{2 p q+p-q-2}{4 p q-p-2 q-1},-\frac{p-2}{1+p}\right]$} & {$\left[-\frac{q_{1}-2}{q_{1}+1},-\frac{p_{1}-q_{1}}{\left(2 p_{1}-1\right)\left(q_{1}-1\right)}\right]$} \\
\hline $01 / 01$ & $\overrightarrow{a_{0} a_{1}^{\prime}}=\overrightarrow{a_{0}^{\prime \prime} a_{1}^{\prime \prime}}$ & {$\left[\frac{4 p q-2 p-q-1}{2 p q-p+q-2},-\frac{q+1}{q-2}\right]$} & {$\left[\frac{p_{1}-q_{1}}{\left(p_{1}-2\right)\left(q_{1}-1\right)}, \frac{2 q_{1}-1}{q_{1}+1}\right]$} \\
\hline $02 / 01$ & $\overrightarrow{a_{0} a_{2}}=\overrightarrow{a_{0}^{\prime \prime} a_{1}^{\prime \prime}}$ & {$\left[\frac{p+2 q-3}{2 p+q-3}, \frac{3 p q-2 p-q}{3 p q-p-2 q}\right]$} & {$\left[\frac{\left(p_{1}-1\right)\left(2 q_{1}-1\right)}{\left(2 p_{1}-1\right)\left(q_{1}-1\right)} \frac{\left(p_{1}-1\right)\left(q_{1}-2\right)}{\left(p_{1}-2\right)\left(q_{1}-1\right)}\right]$} \\
\hline $00 / 12$ & $\overrightarrow{a_{0} a_{0}}=\overrightarrow{a_{1}^{\prime \prime} a_{2}^{\prime \prime}}$ & {$\left[-\frac{(p-2)(q-1)}{p q+2 p+q-4}, \frac{(2 p-1)(q-1)}{4 p q-p-2 q-1}\right]$} & {$\left[\frac{3 p_{1} q_{1}-p_{1}-2 q_{1}}{3 p_{1} q_{1}-2 p_{1}-q_{1}}, \frac{\left(2 p_{1}-1\right)\left(q_{1}-1\right)}{\left(p_{1}-1\right)\left(2 q_{1}-1\right)}\right]$} \\
\hline $00 / 20$ & $\overrightarrow{a_{0} a_{0}}=\overrightarrow{a_{2}^{\prime \prime} a_{0}^{\prime \prime}}$ & {$\left[\frac{2 p-1}{p+1}, \frac{2 p q+p-q-2}{p q+2 p+q-4}\right]$} & {$\left[-\frac{p_{1}+1}{p_{1}-2}, \frac{\left(p_{1}-1\right)\left(2 q_{1}-1\right)}{p_{1}-q_{1}}\right]$} \\
\hline
\end{tabular}

Example 4.11. In Example 2.7, we identified Hajja's $s$-median operator $\mathcal{H}_{s}$ with $\mathcal{M}_{0,1-s}^{00 / 12}$ for $s \in \mathbb{R}$. The above formula (4.10) (cf. Table 1) translates it as

$$
\mathcal{H}_{s}=\mathcal{M}_{0,1-s}^{00 / 12}=\mathcal{S}_{\frac{2 s}{s+3}, \frac{s}{2 s-3}} .
$$

The last expression for $s=-3, \frac{3}{2}$ appears to be singular as $\mathcal{S}_{\infty, \frac{1}{3}}, \mathcal{S}_{\frac{2}{3}, \infty}$ respectively, but these singularities can be removed in the language of $\left(\eta, \eta^{\prime}\right)$-parameters: Indeed, by $(3.2)$ we can interpret $\mathcal{S}_{0,1-s}=\mathcal{S}\left[s \omega+(1-s) \omega^{2}, s \omega^{2}+(1-s) \omega\right]$, hence from Definition 3.6, we obtain $\mathcal{M}_{0,1-s}^{00 / 12}=\mathcal{M}^{00 / 12}\left[s \omega+(1-s) \omega^{2}, s \omega^{2}+(1-s) \omega\right]$. Corollary 3.8 then allows us to compute

$$
\begin{aligned}
\mathcal{H}_{s} & =\mathcal{M}^{00 / 12}\left[s \omega+(1-s) \omega^{2}, s \omega^{2}+(1-s) \omega\right] \\
& =\mathcal{S}\left[s+\omega, s+\omega^{2}\right]=J^{2}+s\left(\frac{2}{3} I-\frac{1}{3} \mathrm{~J}-\frac{1}{3} J^{2}\right)
\end{aligned}
$$


which makes senses on all $s \in \mathbb{C}$. Finally, formulas (4.1)- (4.5) transform $\mathcal{H}_{s}$ into various expressions of generalized medians. For example, for generic complex parameter $s$, one has:

$$
\mathcal{H}_{s}=\mathcal{M}_{\frac{s-2}{s-1}, \frac{s}{s-1}}^{00 / 01}=\mathcal{M}_{\frac{s}{2}, \frac{1}{s-1}}^{01 / 01}=\mathcal{M}_{\frac{1}{1-s}, \frac{2-s}{2}}^{02 / 01} .
$$

Example 4.15 (Parameters for $\mathcal{M}_{p, q}^{\mathrm{wx} / \mathrm{yz}}=\mathcal{S}_{p, q}$ ). Let wx/yz be a given label with w, x, y, z $\in$ $\mathbb{Z} / 3 \mathbb{Z}, \mathrm{y} \neq \mathrm{z}$. By Proposition 3.7 , we find that $\mathcal{M}^{\mathrm{wx} / \mathrm{yz}}\left[\eta, \eta^{\prime}\right]=\mathcal{S}\left[\eta, \eta^{\prime}\right]$ has a unique solution in the form

$$
\mathcal{S}\left[\eta, \eta^{\prime}\right]=\left(\mathrm{J}^{\mathrm{x}}+\mathrm{J}^{\mathrm{y}}-\mathrm{J}^{\mathrm{z}}\right)^{-1} \mathrm{~J}^{\mathrm{w}}=\alpha \mathbf{l}+\beta \mathrm{J}+\gamma \mathrm{J}^{2}
$$

summarized in the following table

\begin{tabular}{cc|cc|c|c} 
Label & {$[\alpha, \beta, \gamma]$} & Label $[\alpha, \beta, \gamma]$ & Label $[\alpha, \beta, \gamma]$ \\
\hline $00 / 01$ & {$[4 / 7,2 / 7,1 / 7]$} & $01 / 01[1,0,0]$ & $02 / 01[1 / 2,1 / 2,0]$ \\
$00 / 10[0,0,1]$ & $01 / 10[1 / 7,2 / 7,4 / 7]$ & $02 / 10[0,1 / 2,1 / 2]$ \\
$00 / 02[4 / 7,1 / 7,2 / 7]$ & $01 / 02[1 / 2,0,1 / 2]$ & $02 / 02[1,0,0]$ \\
$00 / 20[0,1,0]$ & $01 / 20[0,1 / 2,1 / 2]$ & $02 / 20[1 / 7,4 / 7,2 / 7]$ \\
$00 / 12[1 / 2,0,1 / 2]$ & $01 / 12[2 / 7,1 / 7,4 / 7]$ & $02 / 12[0,0,1]$ \\
$00 / 21[1 / 2,1 / 2,0]$ & $01 / 21[0,1,0]$ & $02 / 21[2 / 7,4 / 7,1 / 7]$
\end{tabular}

Recall from Proposition 3.5 (ii) that the corresponding parameter $\left(\eta, \eta^{\prime}\right)$ for each case is given by

$$
\left\{\begin{array}{l}
\eta=\alpha+\beta \omega^{2}+\gamma \omega, \\
\eta^{\prime}=\alpha+\beta \omega+\gamma \omega^{2} .
\end{array}\right.
$$

Next we search parameters $(p, q)$ with $p q \neq 1$ satisfying $\mathcal{M}_{p, q}^{\mathrm{wx} / \mathrm{yz}}=\mathcal{S}_{p, q}$ from the above table. They are classified into the following three kinds:

(i) $\mathcal{S}_{p, q} \in\langle\mathrm{J}\rangle\left(=\left\{\mathrm{I}, \mathrm{J}, \mathrm{J}^{2}\right\}\right)$,

(ii) $\mathcal{S}_{p, q}=\frac{1}{2}\left(\mathrm{~J}^{i}+\mathrm{J}^{j}\right)(i \neq j)$,

$[01 / 01,00 / 10,02 / 02,00 / 20,02 / 12,01 / 21]$

$[02 / 01,02 / 10,01 / 02,01 / 20,00 / 12,00 / 21] ;$

(iii) $\mathcal{S}_{p, q}=\alpha \mathbf{l}+\beta \mathbf{J}+\gamma \mathbf{J}^{2}\left(\{\alpha, \beta, \gamma\}=\left\{\frac{1}{7}, \frac{2}{7}, \frac{4}{7}\right\}\right), \quad[00 / 01,01 / 10,00 / 02,02 / 20,01 / 12,02 / 21]$.

The first two cases are uninteresting: (i) occurs when $(p, q)=(0,0),(1, *),(*, 1)$ (where $* \neq 1$ ) so that $\mathcal{S}_{p, q}$ simply represents a permutation of vertex labels (cf. [8, (3.2)]); (ii) occurs when $\mathcal{S}_{p, q}(\Delta)$ represents the midpoint triangle, while the sides of $\mathcal{M}_{p, q}^{\mathrm{wx} / \mathrm{yz}}(\Delta)$ consists of the half sides of $\Delta$ when $(p, q)=\left(0, \frac{1}{2}\right),\left(\frac{1}{2}, 0\right)$. (Note: $\mathcal{S}_{p, q}=\frac{1}{2}(\mathrm{I}+\mathrm{J})$ never occurs). However, (iii) yields geometrically nontrivial cases (as in Figure 4 when $(p, q)=\left(\frac{4}{5}, \frac{2}{3}\right),\left(\frac{1}{5}, \frac{1}{3}\right),\left(\frac{2}{3}, \frac{1}{3}\right),\left(\frac{1}{3}, \frac{2}{3}\right),\left(\frac{1}{3}, \frac{1}{5}\right),\left(\frac{2}{3}, \frac{4}{5}\right)$. These are operations for Routh's triangles discussed in [8, Example 5.3].

\section{Shape space and Bényi-Ćurgus lifts}

One advantage of considering the (finite) Fourier transforms of triangle triples (3.1) is to enable us to catch directly the shape function

$$
\psi(\Delta):=\frac{\psi_{2}(\Delta)}{\psi_{1}(\Delta)} \in \mathbb{C} \cup\{\infty\}=\mathbf{P}_{\psi}^{1}(\mathbb{C})
$$

for triangle triples $\Delta$ without triple collision, i.e., $\Delta \notin\{(a, a, a) \mid a \in \mathbb{C}\}$. The shape function was explicitly introduced by works of Hajja (e.g. [4]) and has been investigated by many authors including Nicollier [9], Bényi-Ćurgus [1. The idea of applying finite Fourier transformation to study polygon geometry can be traced back to I.J.Schoenberg [10. The value of shape function $\psi(\Delta)$ represents the modulus of shape (similarity class) of $\Delta$ as a triangle with vertices labelled as vector components, whereas the triple power $\psi^{3}(\Delta)$ represents the shape of $\Delta$ as an oriented 


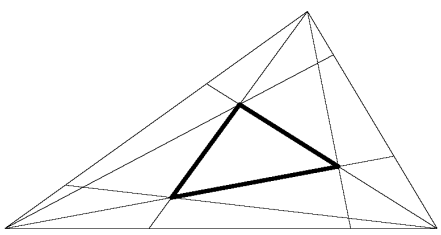

$\mathcal{M}_{\frac{4}{5}, \frac{2}{3}}^{00 / 01}(\Delta)=\mathcal{S}_{\frac{4}{5}, \frac{2}{3}}(\Delta)$

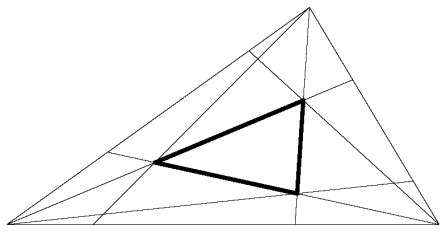

$\mathcal{M}_{\frac{2}{3}, \frac{4}{5}}^{02 / 21}(\Delta)=\mathcal{S}_{\frac{2}{3}, \frac{4}{5}}(\Delta)$

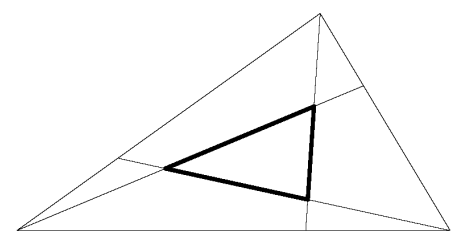

$\mathcal{M}_{\frac{2}{3}, \frac{1}{3}}^{00 / 02}(\Delta)=\mathcal{S}_{\frac{2}{3}, \frac{1}{3}}(\Delta)$

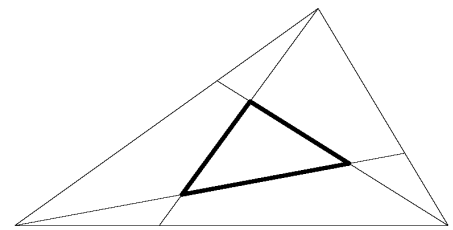

$\mathcal{M}_{\frac{1}{3}, \frac{2}{3}}^{02 / 20}(\Delta)=\mathcal{S}_{\frac{1}{3}, \frac{2}{3}}(\Delta)$

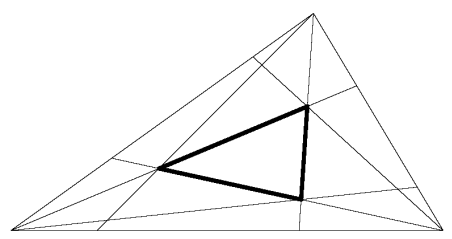

$\mathcal{M}_{\frac{1}{5}, \frac{1}{3}}^{01 / 10}(\Delta)=\mathcal{S}_{\frac{1}{5}, \frac{1}{3}}(\Delta)$

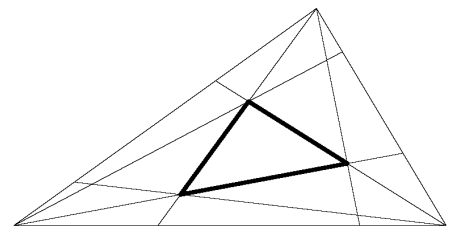

$\mathcal{M}_{\frac{1}{3}, \frac{1}{5}}^{01 / 12}(\Delta)=\mathcal{S}_{\frac{1}{3}, \frac{1}{5}}(\Delta)$

Figure 4. Type (iii) for $\mathcal{M}_{p, q}^{\mathrm{wx} / \mathrm{yz}}(\Delta)=\mathcal{S}_{p, q}(\Delta)$ with $\Delta=\Delta(0)=(0,1,0.7+0.5 i)$

triangle with unlabelled vertices, which is also useful in some geometrical problem (cf. e.g., [7]). For our later discussions in $\S 6$, it is useful to set up the moduli space of triangles (with no triple collision) and their value spaces for $\psi, \psi^{3}$ :

Construction 5.2. Write $\left(\mathbb{C}^{3}\right)^{b}:=\mathbb{C}^{3}-\{(a, a, a) \mid a \in \mathbb{C}\}$ for the collection of triangle triples with no triple collisions, and consider the Fourier transform $\Psi=\left(\psi_{0}, \psi_{1}, \psi_{2}\right)$ of (3.1) as a vector valued function $\left(\mathbb{C}^{3}\right)^{b} \rightarrow \mathbb{C}^{3}$. Noticing that $\psi_{0}(\Delta)$ concerns positioning of (the centroid of) $\Delta$, we may regard the projection to the last two components as the classifying map to the space of translation classes of triangles (written $C^{b}(\psi)$ ) in the form

$$
\operatorname{pr}:\left(\mathbb{C}^{3}\right)^{b} \rightarrow C^{b}(\psi):=\left\{\left(\begin{array}{l}
\psi_{2} \\
\psi_{1}
\end{array}\right) \in \mathbb{C}^{2} \mid\left(\psi_{1}, \psi_{2}\right) \neq(0,0)\right\} .
$$

Here we mean by 'triangle' an ordered triple $(a, b, c) \in \mathbb{C}^{3}$ of three vertices admitting double collisions but no triple collisions. The shape function $\psi(\Delta)=\frac{\psi_{2}(\Delta)}{\psi_{1}(\Delta)}$ for $\Delta \in\left(\mathbb{C}^{3}\right)^{b}$ factors through the space $C^{b}(\psi)$ and terminates in $\mathbf{P}_{\psi}^{1}(\mathbb{C})=\mathbb{C} \cup\{\infty\}$ which is naturally regarded as the moduli space of similarity classes of triangles (with labelled vertices). We call $\mathbf{P}_{\psi}^{1}(\mathbb{C})$ the shape sphere. Denote by $\mathbf{P}_{33}^{1}(\mathbb{C})$ the quotient orbifold of $\mathbf{P}_{\psi}^{1}(\mathbb{C})$ by the multiplication action of the cyclic group $\left\{1, \omega, \omega^{2}\right\}$, where the subscript ' 33 ' indicates the two elliptic points of order 3 at $0, \infty$. Note that the set of complex points $\mathbf{P}_{33}^{1}(\mathbb{C})$ corresponds bijectively to $\left\{\psi(\Delta)^{3} \mid \Delta \in\left(\mathbb{C}^{3}\right)^{b}\right\}$.

The relation of the above four spaces are summarized as in the commutative diagram

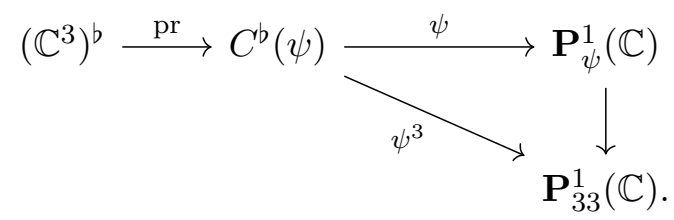

Note 5.4. The space $\mathbf{P}_{\psi}^{1}(\mathbb{C})$ is a complex analytic model of the shape sphere appearing in the study of 3-body problem in celestial dynamics (see, e.g., [6]). 
Since the operator $\mathcal{S}\left[\eta, \eta^{\prime}\right]$ acts on $\Delta$ with componentwise multiplication by $\left(1, \eta^{\prime}, \eta\right)$ to $\Psi(\Delta)$ (cf. (3.3)), it acts on the values of shape function $\psi(\Delta)=\psi_{2}(\Delta) / \psi_{1}(\Delta)$ as

$$
\psi\left(\mathcal{S}\left[\eta, \eta^{\prime}\right](\Delta)\right)=\left(\frac{\eta}{\eta^{\prime}}\right) \psi(\Delta) .
$$

In Example 2.7, we discussed Hajja's operator $\mathcal{H}_{s}$ as a special case of generalized median operator $\mathcal{M}_{0,1-s}^{00 / 12}$. As shown in Example 4.11, it is written in Fourier parameters $\left(\eta, \eta^{\prime}\right)$ as

$$
\mathcal{H}_{s}=\mathcal{M}_{0,1-s}^{00 / 12}=\mathcal{S}\left[s+\omega, s+\omega^{2}\right]=W\left(\begin{array}{ccc}
1 & 0 & 0 \\
0 & s+\omega^{2} & 0 \\
0 & 0 & s+\omega
\end{array}\right) W^{-1} .
$$

On the other hand, Bényi-Ćurgus [1] introduces an operator ' $\mathcal{C}_{s}$ ' (called the binary Ceva operator after a seminal article [3]) for a real parameter $s$ closely related to $\mathcal{H}_{s}$. Although their formulation is given in slightly different language of side length triples, we may extend the equivalent notion for complex parameter $s \in \mathbb{C}$ as follows.

Definition 5.7 (binary Ceva operator). For complex $s \in \mathbb{C}$, define the operator $\mathcal{C}_{s}$ on triangle triples by the following matrix expression:

$$
\mathcal{C}_{s}=W\left(\begin{array}{ccc}
1 & 0 & 0 \\
0 & 0 & s+\omega \\
0 & s+\omega^{2} & 0
\end{array}\right) W^{-1} .
$$

In other words, $\mathcal{C}_{s}$ is defined so as to operate on the Fourier parameters of triangle triples by:

$$
\psi_{0}\left(\mathcal{C}_{s}(\Delta)\right)=\psi_{0}(\Delta), \quad \psi_{1}\left(\mathcal{C}_{s}(\Delta)\right)=(s+\omega) \psi_{2}(\Delta), \quad \psi_{2}\left(\mathcal{C}_{s}(\Delta)\right)=\left(s+\omega^{2}\right) \psi_{1}(\Delta) .
$$

It is not difficult to see that either of $\mathcal{H}_{s}, \mathcal{C}_{s}$ preserve $\left(\mathbb{C}^{3}\right)^{b} \subset \mathbb{C}^{3}$ if and only if $s \neq \rho, \rho^{-1}$. Assuming this condition, let us write $\overline{\mathcal{H}}_{s}, \overline{\mathcal{C}}_{s}$ for the operators on $C^{b}(\psi)$ induced respectively from $\mathcal{H}_{s}, \mathcal{C}_{s}$. These are also expressed as matrices acting on $\left(\begin{array}{l}\psi_{2} \\ \psi_{1}\end{array}\right) \in C^{b}(\psi)$ in the form

$$
\overline{\mathcal{H}}_{s}=\left(\begin{array}{cc}
s+\omega & 0 \\
0 & s+\omega^{2}
\end{array}\right), \quad \overline{\mathcal{C}}_{s}=\left(\begin{array}{cc}
0 & s+\omega^{2} \\
s+\omega & 0
\end{array}\right) .
$$

Below we use the quantity $\xi_{s}:=\frac{s+\omega}{s+\omega^{2}}$ to denote the multiplier factor for $\mathcal{H}_{s}$ on the shape function. Note that the condition $s \neq \rho, \rho^{-1}$ is equivalent to $\xi_{s} \in \mathbb{C}^{\times}$. The following numerical identities easily derived from definitions relate the shapes of $\Delta, \mathcal{H}_{s}(\Delta), \mathcal{C}_{s}(\Delta), \mathcal{H}_{1-s}(\Delta)$ and of $\mathcal{C}_{1-s}(\Delta):$

$$
\psi\left(\mathcal{H}_{s}(\Delta)\right)=\xi_{s} \cdot \psi(\Delta), \quad \psi\left(\mathcal{C}_{s}(\Delta)\right)=\xi_{s}^{-1} \psi(\Delta)^{-1}, \quad \xi_{s}^{-1}=\xi_{1-s} \quad\left(s \neq \rho, \rho^{-1}\right) .
$$

We are then led to Proposition 5.11 below which translates selected geometrical relations found by Bényi-Curgus [1] into our language of operations $\mathcal{H}_{s}, \mathcal{C}_{s}$. We recall that two triangles $\Delta=$ $(a, b, c)$ and $\Delta^{\prime}=\left(a^{\prime}, b^{\prime}, c^{\prime}\right)$ are called directly similar (resp. reversely similar) in [1, p.378] if $\left(a^{\prime}, b^{\prime}, c^{\prime}\right)$ is similar to $(a, b, c)$ (resp. $\left.(a, c, b)\right)$ as oriented triangles without labeling of vertices. We will write $\Delta \stackrel{\text { dr }}{\sim} \Delta^{\prime}$ (resp. $\Delta \stackrel{\text { rv }}{\sim} \Delta^{\prime}$ ) for the direct similarity (resp. reverse similarity) which is equivalent to $\psi(\Delta)^{3}=\psi\left(\Delta^{\prime}\right)^{3}$ (resp. $\psi(\Delta)^{3}=\psi\left(\Delta^{\prime}\right)^{-3}$ ).

Proposition 5.11. Notations being as above, the following formulas and statements hold for $s, r, u \in \mathbb{C} \backslash\left\{\rho, \rho^{-1}\right\}$.

(i) $\overline{\mathcal{H}}_{s} \circ \overline{\mathcal{H}}_{s}=-\overline{\mathcal{C}}_{1-s} \circ \overline{\mathcal{C}}_{s}$.

(ii) $\overline{\mathcal{C}}_{s} \circ \overline{\mathcal{C}}_{s}=\left(s^{2}-s+1\right)$ id.

(iii) For each $\Delta \in\left(\mathbb{C}^{3}\right)^{b}$, we have $\mathcal{C}_{s}(\Delta) \stackrel{\text { rv }}{\sim} \mathcal{H}_{s}(\Delta)$. 
(iv) For each $\Delta \in\left(\mathbb{C}^{3}\right)^{b}, \mathcal{C}_{s} \circ \mathcal{C}_{r}(\Delta) \stackrel{\text { dr }}{\sim} \mathcal{C}_{u}(\Delta)$ if and only if $\left(\xi_{r} \xi_{u}\right)^{3} \cdot \psi(\Delta)^{6}=\xi_{s}^{3}$.

(v) $\mathcal{C}_{s} \circ \mathcal{C}_{r}(\Delta) \stackrel{\text { rv }}{\sim} \mathcal{C}_{u}(\Delta)$ for all $\Delta \in\left(\mathbb{C}^{3}\right)^{b}$ if and only if $\left(\xi_{s} \xi_{u}\right)^{3}=\xi_{r}^{3}$.

(vi) If $\Delta \stackrel{\text { rv }}{\sim} \Delta^{\prime}$, then $\mathcal{C}_{s}(\Delta) \stackrel{\text { rv }}{\sim} \mathcal{C}_{1-s}\left(\Delta^{\prime}\right)$.

Proof. (i), (ii) follow from matrix computations by (5.9), and (iii) follows from (5.10) at once. The values of shape function at $\mathcal{C}_{s} \circ \mathcal{C}_{r}(\Delta)$ and at $\mathcal{C}_{u}(\Delta)$ are respectively $\xi_{s}^{-1} \xi_{r} \cdot \psi(\Delta)$ and $\xi_{u}^{-1} \psi(\Delta)^{-1}$. This proves (iv). Next, by a remark preceding the proposition, $\mathcal{C}_{s} \circ \mathcal{C}_{r}(\Delta)$ is reversely similar to $\mathcal{C}_{u}(\Delta)$ iff $\left(\xi_{s}^{-1} \xi_{r} \psi(\Delta)\right)^{3}=\left(\xi_{u}^{-1} \psi(\Delta)^{-1}\right)^{-3}$, from which the assertion (v) follows by cancelling out the common factor $\psi(\Delta)^{3}$. Finally, the assumption of (vi) is equivalent to $\psi(\Delta)^{3} \psi\left(\Delta^{\prime}\right)^{3}=1$. On the other hand, the entry quotients of the identities $\overline{\mathcal{C}}_{s}(\Delta)=$ $\left(\begin{array}{c}\left(s+\omega^{2}\right) \psi_{1}(\Delta) \\ (s+\omega) \psi_{2}(\Delta)\end{array}\right)$ and $\overline{\mathcal{C}}_{1-s}\left(\Delta^{\prime}\right)=\left(\begin{array}{c}(-s-\omega) \psi_{1}\left(\Delta^{\prime}\right) \\ \left(-s-\omega^{2}\right) \psi_{2}\left(\Delta^{\prime}\right)\end{array}\right)$ yield $\psi\left(\mathcal{C}_{s}(\Delta)\right) \cdot \psi\left(\mathcal{C}_{1-s}\left(\Delta^{\prime}\right)\right)=\psi(\Delta)^{-1} \psi\left(\Delta^{\prime}\right)^{-1}$. Thus, we conclude $\psi\left(\mathcal{C}_{s}(\Delta)\right)^{3} \psi\left(\mathcal{C}_{1-s}\left(\Delta^{\prime}\right)\right)^{3}=1$, that is, $\mathcal{C}_{s}(\Delta) \stackrel{\text { rv }}{\sim} \mathcal{C}_{1-s}\left(\Delta^{\prime}\right)$ as desired.

Note 5.12. The assertions (i), (ii), (iv), (v) and (vi) of Proposition 5.11 correspond respectively to a property at line -5 in p.379, Proposition 9.2, Corollary 9.8, Theorem 9.9 and Proposition 9.1 of [1]. The assertion (iii) is originally a source defining property for the binary Ceva operator [1, p.379]. A binary operation $*$ defined by the identity $\xi_{s} \xi_{s^{\prime}}=\xi_{s * s^{\prime}}$ gives a commutative group structure on $\left\{s \in \mathbf{P}^{1}(\mathbb{C}) \mid s \neq \rho, \rho^{-1}\right\}$ which is equivalent to an operation $\square$ on $\mathbb{R} \cup\{\infty\}$ introduced in [1] and to the additive operation $[+]$ on $\mathbf{P}^{1}(\mathbb{C})-\left\{\rho, \rho^{-1}\right\}$ studied in [8] after suitable variable changes.

Note 5.13. Proposition 5.11 (i), (ii) can be rephrased at the level of operators on $\left(\mathbb{C}^{3}\right)^{b}$ respectively as:

(i) $\mathcal{H}_{s} \circ \mathcal{H}_{s}=\mathcal{S}[-1,-1] \circ \mathcal{C}_{1-s} \circ \mathcal{C}_{s}$

(ii) $\mathcal{C}_{s} \circ \mathcal{C}_{s}=\mathcal{S}\left[s^{2}-s+1, s^{2}-s+1\right]$.

Note 5.14. The above usage of 'reverse similarity' differs from the one employed in [7] where it meant $\Delta \stackrel{\text { dr }}{\sim} \overline{\Delta^{\prime}}$ (mirror image of $\Delta^{\prime}$ ) that is sometimes called anti-similarity.

\section{Tracing orbits of triangles}

Since our operators $\mathcal{S}\left[\eta, \eta^{\prime}\right]$ are realized as linear actions on $\mathbb{C}^{3}$ that fix $(1,1,1)$ by Proposition 3.5 (i), they commute with every complex affine transformation of triangles, in other words, $\mathcal{S}\left[\eta, \eta^{\prime}\right]$ commutes with any mapping of the form $(a, b, c) \mapsto(f(a), f(b), f(c))$ where $f: z \mapsto \lambda z+\nu$ $(\lambda, \nu \in \mathbb{C})$. This is not always the case for real affine transformations. We first begin with the following simple lemma.

Lemma 6.1. Let $\left(\eta, \eta^{\prime}\right) \in \mathbb{C}^{2}$. The operation $\mathcal{S}\left[\eta, \eta^{\prime}\right]$ commutes with the real affine transformations of triangles if and only if $\bar{\eta}=\eta^{\prime}$, i.e., $\eta$ and $\eta^{\prime}$ are complex conjugate to each other.

Proof. Recall that any real affine transformation of the complex plane $\mathbb{C}$ can be written as $f_{\lambda, \mu, \nu}(z)=\lambda z+\mu \bar{z}+\nu$ with $\lambda, \mu, \nu \in \mathbb{C}$. Given a triangle triple $\Delta=(a, b, c)$ and $f=f_{\lambda, \mu, \nu}$, write $f(\Delta):=(f(a), f(b), f(c))$ for the image of $\Delta$ by $f$. Then, one computes

$$
\mathcal{S}\left[\eta, \eta^{\prime}\right]\left(f_{\lambda, \mu, \nu}\left(\begin{array}{l}
a \\
b \\
c
\end{array}\right)\right)=f_{\lambda, \mu, \nu}\left(\mathcal{S}\left[\eta, \eta^{\prime}\right]\left(\begin{array}{l}
a \\
b \\
c
\end{array}\right)\right)+\mu \cdot\left(\mathcal{S}\left[\eta, \eta^{\prime}\right]-\overline{\mathcal{S}\left[\eta, \eta^{\prime}\right]}\right)\left(\begin{array}{l}
a \\
b \\
c
\end{array}\right) .
$$

The commutativity of $\mathcal{S}\left[\eta, \eta^{\prime}\right]$ and $f_{\lambda, \mu, \nu}$ holds if and only if $\mu=0$ (i.e., $f_{\lambda, \mu, \nu}$ is complex affine) or $\mathcal{S}\left[\eta, \eta^{\prime}\right]=\frac{1}{3}\left(1+\eta+\eta^{\prime}\right) \mathbf{I}+\frac{1}{3}\left(1+\eta \omega+\eta^{\prime} \omega^{2}\right) \mathrm{J}+\frac{1}{3}\left(1+\eta \omega^{2}+\eta^{\prime} \omega\right) \mathrm{J}^{2}$ is in $M_{3}(\mathbb{R})$. The latter condition is easily seen to be equivalent to $\bar{\eta}=\eta^{\prime}$.

In [8], we called $\mathcal{S}\left[\eta, \eta^{\prime}\right] \in \mathbb{S}$ an area-preserving operator if the associated parameters $\eta, \eta^{\prime} \in \mathbb{C}$ satisfy $|\eta|=\left|\eta^{\prime}\right|=1$. The set of area-preserving operators forms a compact multiplicative torus 
in $\mathrm{GL}_{3}(\mathbb{C})$. Since $\mathcal{S}\left[\eta, \eta^{\prime}\right]^{k}=\mathcal{S}\left[\eta^{k}, \eta^{\prime k}\right](k \in \mathbb{Z})$, iteration of area-preserving operators can be interpolated by one-parameter family of the form

$$
\left\{\mathcal{S}\left[e^{2 \pi i m t}, e^{2 \pi i n t}\right]\right\}_{t \in \mathbb{R}}
$$

We are particularly interested in the case where three vertices move along a single closed orbit cyclically replacing positions of each other after $t \mapsto t+\varpi / 3$ so that the total motion is left invariant after $t \mapsto t+\varpi$. Note that, in this situation, we may assume $\varpi=1$ and $m, n$ are coprime integers without loss of generality. Taking this into accounts, we are led to start with a more general setup: Recall from 5.2 that $\left(\mathbb{C}^{3}\right)^{b}$ denotes the collection of triangle triples with no triple collisions. Suppose we are given $\Delta \in\left(\mathbb{C}^{3}\right)^{b}$ and two continuous functions $\eta, \eta^{\prime}: \mathbb{R} \rightarrow(\mathbb{R} / \mathbb{Z} \rightarrow) \mathbb{C}$ (with period 1 ). We shall consider the periodic maps $\mathbb{R} \rightarrow \mathbb{R} / \mathbb{Z} \rightarrow\left(\mathbb{C}^{3}\right)^{b}$ in the form

$$
\Delta(t)=\mathcal{S}\left[\eta(t), \eta^{\prime}(t)\right](\Delta) \text { or } \mathcal{M}^{\mathrm{wx} / \mathrm{yz}}\left[\eta(t), \eta^{\prime}(t)\right](\Delta) .
$$

Note that generally $\Delta(0)$ may not be the same as the initial $\Delta$ and that $\Delta(t)$ may degenerate at some $t$ even if $\Delta$ is given as a non-degenerate triangle. The family $\{\Delta(t)\}_{t}$ will be called collision-free if, for every $t \in \mathbb{R}, \Delta(t)$ is a (degenerate or non-degenerate) triangle with three distinct vertices. We sometimes regard the time parameter $t \in \mathbb{R}$ also as $t \in \mathbb{R} / \mathbb{Z}$ when no confusion could occur.

Definition 6.2. Notations being as above, we say the family $\{\Delta(t)\}_{t \in \mathbb{R} / \mathbb{Z}}$ to have a single tracing orbit in ascending (resp. descending) order, if $\mathrm{J} \Delta(t)=\Delta\left(t+\frac{1}{3}\right)\left(\right.$ resp. $\left.=\Delta\left(t-\frac{1}{3}\right)\right)$.

If $\{\Delta(t)\}_{t}$ has a single tracing orbit in ascending order, and $\Delta(t)$ is written as $\left(a_{0}(t), a_{1}(t), a_{2}(t)\right)$, then, $a_{0}(t)=a_{2}\left(t+\frac{1}{3}\right)=a_{1}\left(t+\frac{2}{3}\right)=a_{0}(t+1)$ for all $t \in \mathbb{R}$. We may interpret a collision-free family with this property as a motion of three particles $a_{0}, a_{1}, a_{2}$ moving along a single closed orbit so that they trace each other chronologically with $a_{0} \rightarrow a_{1} \rightarrow a_{2} \rightarrow a_{0}$.

Proposition 6.3. Let $\Delta \in\left(\mathbb{C}^{3}\right)^{b}$ and $\eta, \eta^{\prime}: \mathbb{R} / \mathbb{Z} \rightarrow \mathbb{C}$ be continuous functions with period 1 .

(i) $\left\{\mathcal{S}\left[\eta(t), \eta^{\prime}(t)\right](\Delta)\right\}_{t}$ has a single tracing orbit in ascending (resp. descending) order if and only if

$$
\begin{gathered}
\eta\left(t+\frac{1}{3}\right)=\eta(t) \omega^{-1}, \eta^{\prime}\left(t+\frac{1}{3}\right)=\eta^{\prime}(t) \omega \quad(t \in \mathbb{R}) \\
\left(\text { resp. } \eta\left(t-\frac{1}{3}\right)=\eta(t) \omega^{-1}, \eta^{\prime}\left(t-\frac{1}{3}\right)=\eta^{\prime}(t) \omega \quad(t \in \mathbb{R})\right)
\end{gathered}
$$

holds.

(ii) Let $\mathrm{wx} / \mathrm{yz}$ be a label for generalized median operators. Then, $\left\{\mathcal{M}^{\mathrm{wx} / \mathrm{yz}}\left[\eta(t), \eta^{\prime}(t)\right](\Delta)\right\}_{t}$ has a single tracing orbit in ascending (resp. descending) order if and only if $\tilde{\eta}(t):=$ $\eta(t)-\omega^{\mathrm{x}-\mathrm{w}}, \tilde{\eta}^{\prime}(t):=\eta^{\prime}(t)-\omega^{\mathrm{w}-\mathrm{x}}$ satisfy

$$
\begin{gathered}
\tilde{\eta}\left(t+\frac{1}{3}\right)=\tilde{\eta}(t) \omega^{-1}, \tilde{\eta}^{\prime}\left(t+\frac{1}{3}\right)=\tilde{\eta}^{\prime}(t) \omega \quad(t \in \mathbb{R}) . \\
\left(\operatorname{resp.} \tilde{\eta}\left(t-\frac{1}{3}\right)=\tilde{\eta}(t) \omega^{-1}, \tilde{\eta}^{\prime}\left(t-\frac{1}{3}\right)=\tilde{\eta}^{\prime}(t) \omega \quad(t \in \mathbb{R}) .\right)
\end{gathered}
$$

Proof. (i) follows immediately from (4.1). To prove (ii), we make use of Corollary 3.8 to express $\mathcal{M}^{\mathrm{wx} / \mathrm{yz}}\left[\eta(t), \eta^{\prime}(t)\right](\Delta)$ as $\mathcal{S}\left[\eta_{0}(t), \eta_{1}(t)\right]$. Then, apply (i) for the latter form.

Let us look more closely at the tracing orbit in relation with the shape sphere $\mathbf{P}_{\psi}^{1}(\mathbb{C})$ introduced in Definition 5.2. Write $\operatorname{Conf}^{3}(\mathbb{C})$ (called the configuration space) for the collection of 
collision-free triples in $\left(\mathbb{C}^{3}\right)^{b}$, i.e.,

$$
\operatorname{Conf}^{3}(\mathbb{C}):=\left\{(a, b, c) \in \mathbb{C}^{3} \mid a \neq b \neq c \neq a\right\} \subset\left(\mathbb{C}^{3}\right)^{b} .
$$

Then, it is easy to see that the shape function $\psi=\frac{\psi_{2}}{\psi_{1}}$ maps $\operatorname{Conf}^{3}(\mathbb{C})$ onto the open locus $\mathbf{P}_{\psi}^{1}(\mathbb{C})-\left\{1, \omega, \omega^{2}\right\}$ of the shape sphere $\mathbf{P}_{\psi}^{1}(\mathbb{C})$. Given a collision-free single tracing orbit $\{\Delta(t)\}_{t}$ $\subset \operatorname{Conf}^{3}(\mathbb{C})$ either in ascending or descending order, the image $\{\psi(\Delta(t))\}_{0 \leq t \leq 1}$ move on a closed curve on $\mathbf{P}_{\psi}^{1}(\mathbb{C})-\left\{1, \omega, \omega^{2}\right\}$. More precisely, during the process starting/ending at the point $\psi_{0}:=\psi(\Delta(0))=\psi(\Delta(1))$, it passes two distinguished points $\psi\left(\Delta\left(\frac{1}{3}\right)\right)=\omega \psi_{0}, \psi\left(\Delta\left(\frac{2}{3}\right)\right)=$ $\omega^{2} \psi_{0}$ (resp. $\left.\psi\left(\Delta\left(\frac{1}{3}\right)\right)=\omega^{2} \psi_{0}, \psi\left(\Delta\left(\frac{2}{3}\right)\right)=\omega \psi_{0}\right)$ if $\{\Delta(t)\}_{t}$ moves in ascending order (resp. in descending order). Thus, in view of the diagram $\sqrt{5.3}$, the image of $\left\{\psi(\Delta(t))^{3}\right\}_{0 \leq t \leq \frac{1}{3}}$ forms a closed curve on $\mathbf{P}_{33}^{1}(\mathbb{C})-\{1\}$. It is worth noting that the (existence and) classification of the tracing orbits $\{\Delta(t)\}_{t}$ sharing a same closed curve on $\mathbf{P}_{33}^{1}(\mathbb{C})-\{1\}$ is available as below, where we employ the convention $1 / 0=\infty$.

Proposition 6.4. Let $\varepsilon \in\{ \pm 1\}$ and let $\gamma: \mathbb{R} \rightarrow \mathbf{P}_{\psi}^{1}(\mathbb{C})-\left\{1, \omega, \omega^{2}\right\}$ be a non-constant continuous map such that $\gamma(0) \notin\{0, \infty\}$ and $\gamma\left(t+\frac{1}{3}\right)=\omega^{\varepsilon} \gamma(t)$ for all $t \in \mathbb{R}$. Set $\xi(t):=\gamma(t) / \gamma(0)$.

(i) There exists a collision-free single tracing orbit $\{\Delta(t)\}_{t}$ with $\psi(\Delta(t))=\gamma(t)$ in ascending (resp. descending) order when $\varepsilon=1$ (resp. $\varepsilon=-1$ ), if and only if $\xi(t)$ can be written in the form $\xi(t)=\frac{\eta_{2}(t)}{\eta_{1}(t)}$ with $\eta_{r}: \mathbb{R} \rightarrow \mathbb{C}$ satisfying $\eta_{r}\left(t+\frac{1}{3}\right)=\omega^{\varepsilon r} \eta_{r}(t)(r=1,2)$.

(ii) In particular, if $\gamma(t) \neq \infty(\forall t)$ then (i) is the case with $\eta_{2}(t)=e^{2 \pi i \varepsilon t} \xi(t), \eta_{1}(t)=e^{2 \pi i \varepsilon t}$.

(iii) Suppose (i) is the case. Then, every possible tracing orbit $\{\Delta(t)\}_{t}$ sharing the same closed curve $\gamma(t)$ as $\psi(\Delta(t))$ is obtained as

$$
\Delta(t)=W\left(\begin{array}{ccc}
1 & 0 & 0 \\
0 & \eta_{1}(t) \mu(t) & 0 \\
0 & 0 & \eta_{2}(t) \mu(t)
\end{array}\right) W^{-1}\left(\begin{array}{l}
a_{0} \\
b_{0} \\
c_{0}
\end{array}\right)
$$

where $\mu: \mathbb{R} \rightarrow \mathbb{C}^{\times}$is a continuous function with period $\frac{1}{3}$, and $\Delta_{0}=\left(a_{0}, b_{0}, c_{0}\right)$ is a triangle triple with $\psi\left(\Delta_{0}\right)=\gamma(0)$.

Proof. The assumption on $\gamma(0)$ tells that the value $\lambda_{0}:=-\frac{\gamma(0) \omega-1}{\gamma(0)-\omega}$ is contained in $\mathbb{C}-\left\{0,1, \rho^{ \pm 1}\right\}$ so that $\Delta_{0}:=\left(0,1, \lambda_{0}\right) \in \operatorname{Conf}^{3}(\mathbb{C})$ forms a (non-equilateral) triangle triple with $\psi\left(\Delta_{0}\right)=\gamma(0)$.

(i) The 'if'-part is already shown in Proposition 6.3, so we here show the 'only if' part. Suppose a collision-free single tracing orbit $\{\Delta(t)\}_{t}$ exists and consider the behavior of $\psi_{r}(\Delta(t))$ $(r=1,2)$. Since $\gamma(0)=\psi(\Delta(0))=\psi_{2}(\Delta(0)) / \psi_{1}(\Delta(0)) \notin\{0, \infty\}$ by assumption, we can define for each $r \in\{1,2\}$ a function $\eta_{r}: \mathbb{R} \rightarrow \mathbb{C}$ by $\eta_{r}(t):=\psi_{r}(\Delta(t)) / \psi_{r}(\Delta(0))$. This shows that $\Psi(\Delta(t))=W^{-1} \Delta(t)$ is of the form $\operatorname{diag}\left(1, \eta_{1}(t), \eta_{2}(t)\right) \Psi(\Delta(0))$. Then the prescribed property $\Delta\left(t+\frac{\varepsilon}{3}\right)=\mathrm{J} \Delta(t)$ (Definition 6.2 implies the required properties for $\eta_{r}\left(t+\frac{1}{3}\right)(r=1,2)$.

(ii) This is immediate after (i).

(iii) Fix a collision-free tracing orbit $\{\Delta(t)\}_{t}$ with parameter functions $\eta_{1}(t), \eta_{2}(t)$ as in (i), and pick any other such a family $\left\{\Delta^{\prime}(t)\right\}_{t}$ with another set of parameter functions $\eta_{1}^{\prime}(t), \eta_{2}^{\prime}(t)$. Then, $\frac{\eta_{2}(t)}{\eta_{1}(t)}=\xi(t)=\frac{\eta_{2}^{\prime}(t)}{\eta_{1}^{\prime}(t)}$ for all $t \in \mathbb{R}$. Note here that $\xi(t)=0($ resp. $=\infty)$ if and only if $\eta_{1} \eta_{1}^{\prime} \neq 0$, $\eta_{2}=\eta_{2}^{\prime}=0$ (resp. $\eta_{2} \eta_{2}^{\prime} \neq 0, \eta_{1}=\eta_{1}^{\prime}=0$ ). So the continuity of the map $\xi$ of $\mathbb{R}$ into the Riemann sphere $\mathbb{C} \cup\{\infty\}$ enables us to define a continuous function $\mu: \mathbb{R} \rightarrow \mathbb{C}^{\times}$by $\mu(t):=\frac{\eta_{1}^{\prime}}{\eta_{1}}\left(=\frac{\eta_{2}^{\prime}}{\eta_{2}}\right)$ when $\xi(t) \in \mathbb{C}^{\times}, \mu(t):=\frac{\eta_{1}^{\prime}}{\eta_{1}}$ when $\xi(t)=0$ and $\mu(t):=\frac{\eta_{2}^{\prime}}{\eta_{2}}$ when $\xi(t)=\infty$. The periodic property $\mu\left(t+\frac{1}{3}\right)=\mu(t)$ is a consequence of $\eta_{r}\left(t+\frac{1}{3}\right)=\omega^{\varepsilon r} \eta_{r}(t)$ and $\eta_{r}^{\prime}\left(t+\frac{1}{3}\right)=\omega^{\varepsilon r} \eta_{r}(t)^{\prime}(r=1,2)$. 
The vector expression in (iii) follows from the procedure described in the above proof of (i) with $\Delta_{0}=\left(a_{0}, b_{0}, c_{0}\right)=W \operatorname{diag}\left(1, \eta_{1}^{\prime}(0), \eta_{2}^{\prime}(0)\right) W^{-1} \Delta^{\prime}(0)$, that is, $\psi\left(\Delta_{0}\right)=\xi(0) \cdot \psi\left(\Delta^{\prime}(0)\right)=$ $\xi(0) \gamma(0)=\gamma(0)$. This completes the proof.

Now, let us turn back to the area-preserving parameters $\eta(t)=e^{2 \pi i m t}, \eta^{\prime}(t)=e^{2 \pi i n t}$ with coprime integers $m, n \in \mathbb{Z}$ and examine some typical cases.

Example 6.5. Let $\Delta$ be a triangle triple and $m, n$ coprime integers. By Proposition 6.3 (i), the family

$$
\left\{\Delta(t)=\mathcal{S}\left[e^{2 \pi i m t}, e^{2 \pi i n t}\right](\Delta)\right\}_{t \in \mathbb{R}}
$$

has a single tracing orbit if $m+n \equiv 0(\bmod 3)$. The vertices move in ascending (resp. descending) order if $m \equiv 2(\bmod 3)($ resp. $m \equiv 1(\bmod 3))$.

Example 6.6 (Steiner ellipse). The special case $m=-1, n=1$ of Example 6.5 is

$$
\Delta(t)=\mathcal{S}\left[e^{-2 \pi i t}, e^{2 \pi i t}\right](\Delta) .
$$

In this case, starting from $\Delta(0)=\Delta$, the vertices of a triangle move on an ellipse with sides tangent to an interior ellipse (Figure 5). For an easy proof for the case $\Delta$ is non-degenerate, one can apply Lemma 6.1 to deform $\Delta$ to the equilateral triangle $\left(1, \omega, \omega^{2}\right)$ in real affine geometry. If $\Delta=(0,1, u+v \sqrt{-1})$, then the circumscribed ellipse has the following equation in XY-coordinates of $\mathbb{C}$.

$$
v^{2}\left(X-\frac{1+u}{3}\right)^{2}+(v-2 u v)\left(X-\frac{1+u}{3}\right)\left(Y-\frac{v}{3}\right)+\left(1-u+u^{2}\right)\left(Y-\frac{v}{3}\right)^{2}=\frac{v^{2}}{3} .
$$

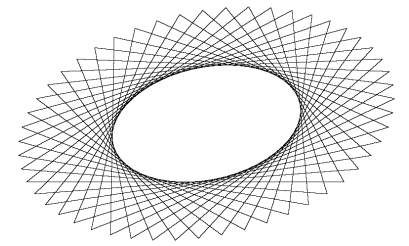

FiguRE 5. $\left\{\mathcal{S}\left[e^{-2 \pi i k / 17}, e^{2 \pi i k / 17}\right](\Delta)\right\}_{k \geq \mathbb{Z}}$ for $\Delta=\Delta(0)=(0,1,0.7+0.5 i)$

Example 6.7. The following three collections of figures (Figure 6, 7, 8) illustrate the family $\mathcal{S}\left[e^{2 \pi i t}, e^{2 \pi i n t}\right](\Delta)$ for $n \equiv 2(\bmod 3), \mathcal{S}\left[e^{2 \pi i m t}, e^{2 \pi i t}\right](\Delta)$ for $m \equiv 2(\bmod 3)$ and some other types from Example 6.5 respectively. We start from $\Delta=\Delta(0)=(0,1,0.7+0.5 i)$.

Example 6.8 (Median orbits). By Proposition 6.3 (ii), the median triangle family

$$
\left\{\mathcal{M}^{0 \mathrm{x} / 01}\left[\eta(t), \eta^{\prime}(t)\right](\Delta)\right\}_{t \in \mathbb{R}}
$$

along with $\eta(t)=e^{2 \pi i m t}+\omega^{\mathrm{x}}, \eta^{\prime}(t)=e^{2 \pi i n t}+\omega^{-\mathrm{x}}(m+n \equiv 0(\bmod 3), \mathrm{x} \in \mathbb{Z} / 3 \mathbb{Z})$ has a single tracing orbit. The following figure (Figure 9 starts from $\Delta=(0,1,0.7+0.5 i), \Delta(0)=$ $\left(\frac{4}{5}+\frac{1}{3} i, \frac{7}{30}+\frac{1}{6} i, \frac{2}{3}\right)$. According to $(4.8)$, the orbit is independent of the choice of $\mathrm{x} \in \mathbb{Z} / 3 \mathbb{Z}$. We also observe that it is similar to the orbit $\left\{\mathcal{S}\left[e^{-10 \pi i t}, e^{4 \pi i t}\right](\Delta)\right\}_{n \in \mathbb{R}}$ illustrated in the previous example.

It is not necessary for us to persist in area-preserving parameters in Proposition 6.3.

Simple linear sums of $e^{2 \pi i m t}$ with $m \equiv \pm 1(\bmod 3)$ ( \pm depends on $\eta, \eta^{\prime}$ individually) already provide us with a number of remarkable examples. In this paper, we content ourselves with showing the following few cases among them. 

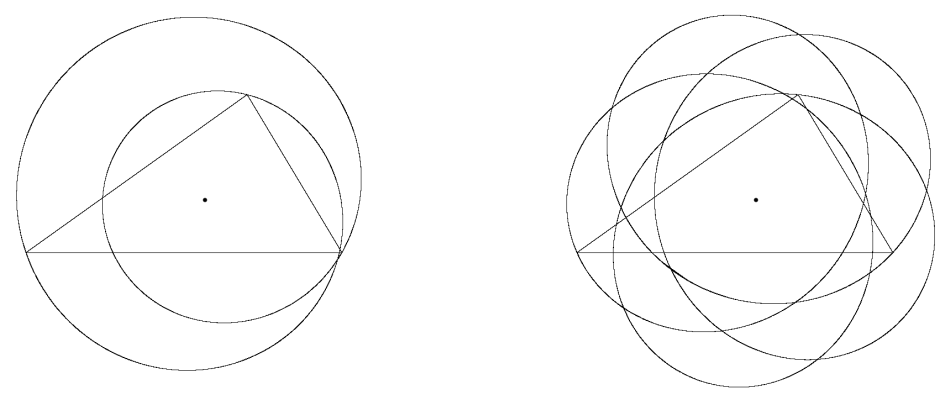

Figure 6. $\left\{\mathcal{S}\left[e^{2 \pi i t}, e^{4 \pi i t},\right](\Delta)\right\}_{t \in \mathbb{R}}$ and $\left\{\mathcal{S}\left[e^{2 \pi i t}, e^{-8 \pi i t}\right](\Delta)\right\}_{t \in \mathbb{R}}$
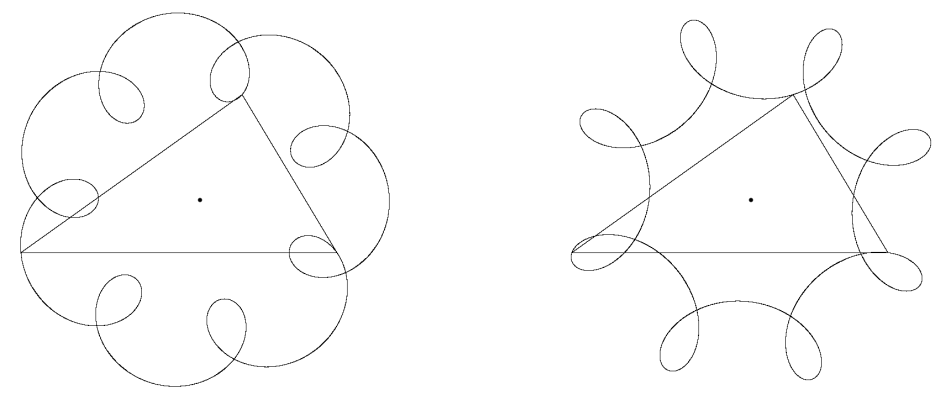

Figure 7. $\left\{\mathcal{S}\left[e^{16 \pi i t}, e^{2 \pi i t}\right](\Delta)\right\}_{t \in \mathbb{R}}$ and $\left\{\mathcal{S}\left[e^{-14 \pi i t}, e^{2 \pi i t}\right](\Delta)\right\}_{t \in \mathbb{R}}$
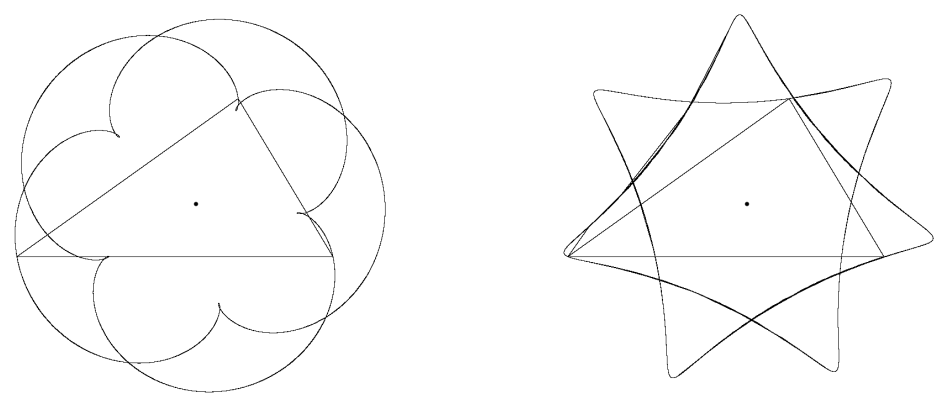

Figure 8. $\left\{\mathcal{S}\left[e^{14 \pi i t}, e^{4 \pi i t}\right](\Delta)\right\}_{t \in \mathbb{R}}$ and $\left\{\mathcal{S}\left[e^{-10 \pi i t}, e^{4 \pi i t}\right](\Delta)\right\}_{t \in \mathbb{R}}$

Example 6.9 (Figure eight cevian orbit). Let $\Delta=(0, i,-i)$ be a degenerate triangle, and set $\eta(t)=-2 e^{2 \pi i t}+e^{-4 \pi i t}, \eta^{\prime}(t)=2 e^{-2 \pi i t}+e^{4 \pi i t}$. Then, the vertices of $\Delta(t)=\mathcal{S}\left[\eta(t), \eta^{\prime}(t)\right](\Delta)$ moves on a single figure eight curve $X^{2}=\frac{3}{16} X^{4}+Y^{2}$ in XY-coordinates of $\mathbb{C}$ : The first vertex of $\Delta(t)$ moves along $\frac{4}{3} \sqrt{3} \cos (t)+i \frac{2}{3} \sqrt{3} \sin (2 t)(t \in \mathbb{R})$ and the other two vertices chase it on the same orbit (Figure 10).

One direction of generalizing this example is to consider $\Delta(t)$ whose vertices move on what is called a Lissajous curve. In a separate article [5], we will discuss in details "Lissajous 3-braids" arising from this sort of triangle's motions. 


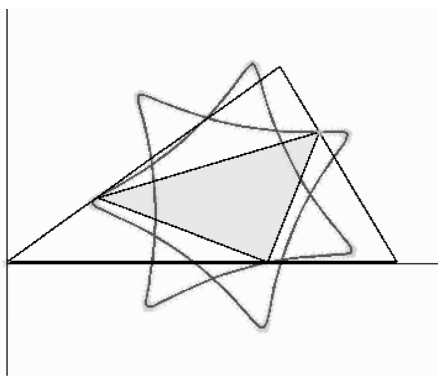

Figure 9. $\Delta$ and $\left\{\mathcal{M}^{0 \mathrm{x} / 01}\left[e^{-10 \pi i t}+\omega^{\mathrm{x}}, e^{4 \pi i t}+\omega^{-\mathrm{x}}\right](\Delta)\right\}_{t \in \mathbb{R}}$
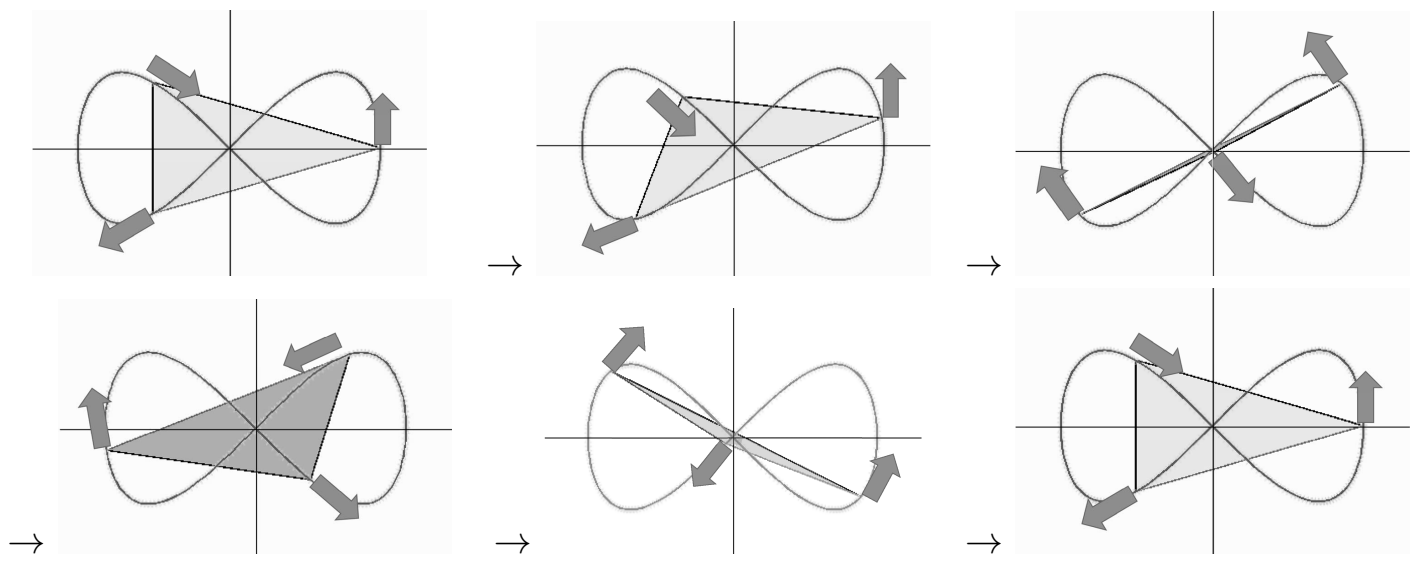

Figure 10. $\left\{\mathcal{S}\left[-2 e^{2 \pi i t}+e^{-4 \pi i t}, 2 e^{-2 \pi i t}+e^{4 \pi i t}\right](\Delta)\right\}_{t \in \mathbb{R}}$

Example 6.10 (Figure eight median orbit). We provide another example for Proposition 6.3 (ii). Starting from $\Delta=(0,4,3+i)$, the median triangle family $\left\{\mathcal{M}^{01 / 01}\left[\eta(t), \eta^{\prime}(t)\right](\Delta)\right\}_{t \in \mathbb{R}}$ along with $\eta(t)=-2 e^{2 \pi i t}+e^{-4 \pi i t}+\omega, \eta^{\prime}(t)=2 e^{-2 \pi i t}+e^{4 \pi i t}+\omega^{2}$ gives a figure eight orbit (Figure 11).
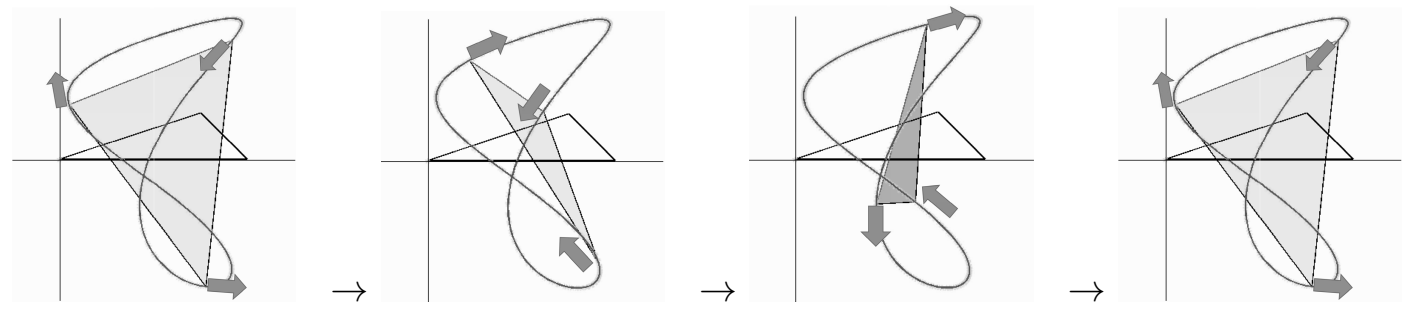

Figure 11. $\Delta$ and $\left\{\mathcal{M}^{01 / 01}\left[-2 e^{2 \pi i t}+e^{-4 \pi i t}+\omega, 2 e^{-2 \pi i t}+e^{4 \pi i t}+\omega^{2}\right](\Delta)\right\}_{t \in \mathbb{R}}$ 


\section{Acknowledgement}

This work was supported by JSPS KAKENHI Grant Numbers JP16K13745, JP20H00115. This is a pre-print of an article published in Results in Mathematics. The final authenticated version is available online at: https://doi.org/s00025-020-01268-3

\section{References}

[1] Á.Bényi, B.Ćurgus, Triangles and groups of cevians, J. of Geometry 103 (2012), 375-408.

[2] R. H. Buchholz, Triangles with Three Rational Medians, J. Number Theory 97 (2002), 113-131; On triangles with rational altitudes, angle bisectors or medians $\mathrm{PhD}$ thesis, University of Newcastle 1989.

[3] H.B.Griffith, Iterated sequences of triangles, Math. Gazette 89, Issue 516 (2005), 518-522.

[4] M. Hajja, On nested sequences of triangles, Result. Math. 54 (2009), 289-299.

[5] E.Kin, H.Nakamura, H.Ogawa, Lissajous 3-braids, preprint. arXiv:2008.00585 [math.GT]

[6] R.Moeckel, R.Montgomery, Realizing all reduced syzygy sequences in the planar three-body problem, Nonlinearity 28 (2015) 1919-1935.

[7] H.Nakamura, K.Oguiso, Elementary moduli spaces of triangles and iterative processes, J. Math. Sci. Univ. Tokyo 10 (2003), 209-224.

[8] H.Nakamura, H.Ogawa, A family of geometric operators on triangles with two complex variables, J. Geom. 111, Article number: 2 (2020), 1-16.

[9] G. Nicollier, Convolution filters for triangles, Forum Geometricorum 13 (2013), 61-85.

[10] I.J.Schoenberg, The finite Fourier series and elementary geometry, Amer. Math. Monthly, 57 (1950), 390-404.

Department of Mathematics, Graduate School of Science, Osaka University, Toyonaka, Osaka 560-0043, JAPAN

E-mail address: nakamura@math.sci.osaka-u.ac.jp

Department of Mathematics, Graduate School of Science, Osaka University, Toyonaka, Osaka 560-0043, JAPAN

E-mail address: ogawa@math.sci.osaka-u.ac.jp 